\title{
Orai1a, but not Orai1 $\beta$, co-localizes with TRPC1 and is required for its plasma membrane location and activation in HeLa cells
}

\author{
Jose Sanchez-Collado ${ }^{1}$. Jose J. Lopez ${ }^{1}$ Isaac Jardin ${ }^{1} \cdot$ Alejandro Berna-Erro ${ }^{1} \cdot$ Pedro J. Camello ${ }^{2}$. \\ Carlos Cantonero ${ }^{1} \cdot$ Tarik Smani $^{3,4}$. Gines M. Salido ${ }^{1} \cdot$ Juan A. Rosado ${ }^{1}$ (i)
}

Received: 29 October 2021 / Revised: 2 December 2021 / Accepted: 15 December 2021 / Published online: 6 January 2022

(c) The Author(s) 2022

\begin{abstract}
The identification of two variants of the canonical pore-forming subunit of the $\mathrm{Ca}^{2+}$ release-activated $\mathrm{Ca}^{2+}(\mathrm{CRAC})$ channel Orai1, Orai $1 \alpha$ and Orai $1 \beta$, in mammalian cells arises the question whether they exhibit different functional characteristics. Orai $1 \alpha$ and Orai1 $\beta$ differ in the N-terminal 63 amino acids, exclusive of Orai1 $\alpha$, and show different sensitivities to $\mathrm{Ca}^{2+}$-dependent inactivation, as well as distinct ability to form arachidonate-regulated channels. We have evaluated the role of both Orai1 variants in the activation of TRPC 1 in HeLa cells. We found that Orai1 $\alpha$ and Orai $1 \beta$ are required for the maintenance of regenerative $\mathrm{Ca}^{2+}$ oscillations, while TRPC1 plays a role in agonist-induced $\mathrm{Ca}^{2+}$ influx but is not essential for $\mathrm{Ca}^{2+}$ oscillations. Using APEX2 proximity labeling, co-immunoprecipitation and the fluorescence of G-GECO1.2 fused to Orai $1 \alpha$ our results indicate that agonist stimulation and $\mathrm{Ca}^{2+}$ store depletion enhance Orai1 $\alpha-$ TRPC 1 interaction. Orai1 $\alpha$ is essential for TRPC1 plasma membrane location and activation. Thus, TRPC1 function in HeLa cells depends on $\mathrm{Ca}^{2+}$ influx through Orai $1 \alpha$ exclusively.
\end{abstract}

Keywords Orai $1 \alpha \cdot$ Orai $1 \beta \cdot$ STIM1 $\cdot$ TRPC $1 \cdot$ Store-operated calcium entry

Jose Sanchez-Collado and Jose J. Lopez contributed equally to this work.

\section{Jose J. Lopez}

jjlopez@unex.es

$\bowtie$ Juan A. Rosado

jarosado@unex.es

1 Department of Physiology (Cellular Physiology Research Group), Institute of Molecular Pathology Biomarkers (IMPB), University of Extremadura, 10003 Caceres, Spain

2 Department of Physiology, (Smooth Muscle Physiology Research Group), Institute of Molecular Pathology Biomarkers, University of Extremadura, 10003 Caceres, Spain

3 Department of Medical Physiology and Biophysics, University of Seville, Seville, Spain

4 Group of Cardiovascular Pathophysiology, Institute of Biomedicine of Seville, University Hospital of Virgen del Rocio/University of Seville/CSIC, Seville, Spain

\section{Introduction}

Agonist-induced changes in cytosolic $\mathrm{Ca}^{2+}$ concentration are finely regulated to ensure the generation of spatiotemporally dynamic $\mathrm{Ca}^{2+}$ signals. The magnitude and pattern of $\mathrm{Ca}^{2+}$ signals depend on the type and concentration of the agonist; thus, low-intensity stimulation leads to oscillatory responses and higher-intensity stimulations generate more sustained responses [1,2]. Store-operated $\mathrm{Ca}^{2+}$ entry (SOCE) is a mechanism for $\mathrm{Ca}^{2+}$ influx involved in a variety of cellular functions including gene transcription and the maintenance of $\mathrm{Ca}^{2+}$ oscillations [3-5]. SOCE is regulated by the filling state of the intracellular $\mathrm{Ca}^{2+}$ stores, primarily the endoplasmic reticulum (ER), so that occupation of phospholipase C-coupled plasma membrane agonist receptors leads to the generation of inositol trisphosphate $\left(\mathrm{IP}_{3}\right)$, which, in turn, induces $\mathrm{Ca}^{2+}$ efflux from the intracellular $\mathrm{Ca}^{2+}$ stores. Store depletion triggers the activation of stromal interaction molecule (STIM) proteins leading to a conformational change that allows the interaction with and activation of Orai channels in the plasma membrane [6-10]. In addition to Orai1, two paralogs have been identified in mammalian cells, Orai2 and Orai3, which hetero-multimerize with Orai1 to fine-tune 
agonist-evoked $\mathrm{Ca}^{2+}$ responses [11]. In addition, two STIM isoforms have been described, STIM1 and STIM2. The Orai and STIM isoforms exhibit differential properties and are non-redundant tailoring-graded $\mathrm{Ca}^{2+}$ signals in response to physiological concentrations of agonists [11-14]. Two storeoperated currents have been identified so far: the well-characterized and highly $\mathrm{Ca}^{2+}$-selective $I_{\text {crac }}$, which involves activation of Orai channels by STIM proteins, and $I_{\text {soc }}$, described in certain cell types, which involves the transient receptor potential (TRP) family member TRPC1, Orai 1 and STIM1 $[15,16]$, that, unlike $I_{\text {crac }}$, is non-selective for cations and exhibits higher conductance[17].

There is substantial evidence that TRPC1 channels are activated by multiple pathways, including receptor-operated $\left(\mathrm{Ca}^{2+}\right.$ store-independent), store-operated (Orai1-dependent) and store-operated (Orai1-independent) mechanisms $[18,19]$. This diversity is likely due to different heteromeric TRPC1 structures [20] and might be cell-specific, for instance, store-mediated activation of TRPC1 is Orai1dependent in salivary glands [21], whereas a store-operated, Orail-independent, TRPC1 activation has been described in vascular smooth muscle cells [22, 23], a pathway that coexists with a mechanism where TRPC 1 functionally interacts with Orai1 and $\mathrm{Ca}_{\mathrm{v}} 1.2$ channels to mediate vasoconstriction [24].

A functional interaction between STIM1, Orai1 and TRPC1 has been reported in several cell types [21, 25]. It has been proposed that depletion of intracellular stores induces STIM1-dependent $\mathrm{Ca}^{2+}$ entry through Orai1 channels, thus leading to a local increase in free $\mathrm{Ca}^{2+}$ concentration that triggers the translocation of TRPC1 to the vicinity of STIM1 and Orai1 in the plasma membrane, where STIM1 can interact with and activate TRPC1 channels [16, 26]. A recent study using single-channel patch clamp experiments has revealed that endogenous TRPC1 forms a channel pore without involving Orai proteins [27] but its activation has been reported to be mostly dependent on Orai1-mediated $\mathrm{Ca}^{2+}$ influx. In this context, TRPC1 activation has been suggested to amplify and/or modify the pattern of Orai1mediated $\mathrm{Ca}^{2+}$ signals [1].

Two Orail variants have been identified in mammalian cells, the long variant, named Orai $1 \alpha$, is the full-length Orai 1 containing 301 amino acids, and the short variant, Orai $1 \beta$ arises from the same transcript by a process of alternative translation initiation from a methionine at position 64 in the Orai1 $\alpha$ variant [28]. Some functional differences have been reported between both Orai1 forms, while both variants can support both $I_{\text {crac }}$ and $I_{\text {soc }}$ with similar efficiencies, only Orai $1 \alpha$ is required for the formation of arachidonate-regulated channels underlying $I_{\text {arc }}$. In addition, Orai $1 \alpha$ is more sensitive to $\mathrm{Ca}^{2+}$-dependent inactivation [15], a mechanism that might involve specific phosphorylation of Orai1 $\alpha$ at Ser34 in an adenylyl cyclase 8-dependent manner [4]. Using different experimental procedures, here we show that Orai $1 \alpha$ functionally interacts with TRPC 1 by a mechanism regulated by agonist stimulation. Functional Orai1 $\alpha$ is essential both for the location of TRPC 1 in the plasma membrane and for cation influx through the channel, by contrast, Orai $1 \beta$, is not required for TRPC1 channel function or plasma membrane expression.

\section{Materials and methods}

\section{Reagents and antibodies}

Fura-2 acetoxymethyl ester (fura-2/AM) was from Molecular Probes (Leiden, The Netherlands). High-glucose Dulbecco's modified Eagle's medium (DMEM), fetal bovine serum, trypsin, penicillin/streptomycin, rabbit polyclonal anti-TRPC1 antibody (catalog number PA577303, epitope: amino acids 557-571 of human TRPC1), mouse monoclonal anti-PMCA antibody (clone 5F10; catalog number MA3-914, epitope: amino acids 724-783 of human PMCA), high-fidelity PCR kit (Platinum ${ }^{\mathrm{TM}}$ SuperFi ${ }^{\mathrm{TM}}$ DNA Polymerase), Clean-Blot ${ }^{\mathrm{TM}}$ IP detection reagent, SuperSignal ${ }^{\circledR}$ West Dura extended duration substrate reagent, Pierce ${ }^{\mathrm{TM}}$ BCA protein assay kit, high-capacity streptavidin agarose resin and EZ-Link ${ }^{\mathrm{TM}}$ Sulfo-NHS-LC-Biotin were purchased from ThermoFisher Scientific (Waltham, MA, USA). Complete EDTA-free protease inhibitor cocktail tablets were from Roche Diagnostics GmbH (Mannheim, Germany). DharmaFECT kb transfection reagent was obtained from Horizon Discovery (Waterbeach, UK). $\mathrm{N}$-Glycosidase F (PNGase F) was purchased from New England Biolabs Inc (Ipswich, MA, USA). Mouse monoclonal Anti-GOK/Stim1 antibody (Clone 44/GOK; catalog number 610954, epitope: amino acids: 25-139 of human STIM1) was purchased from BD Biosciences (San Jose, CA, USA). Thapsigargin (TG), histamine, protein A agarose beads, HEPES (4-(2-Hydroxyethyl)piperazine-1-ethanesulfonic acid), EGTA (ethylene glycol-bis(2-aminoethylether)- $N, N, N^{\prime}, N^{\prime}$-tetraacetic acid), EDTA (ethylenedinitrilotetraacetic acid), bovine serum albumin (BSA), biotin fenol, trolox, sodium azide, sodium ascorbate, biotin, rabbit polyclonal anti-Orail antibody (catalog number O8264, epitope: amino acids 288-301 of human Orai1), rabbit polyclonal anti-Orai1 (AB-1) antibody (catalog number AV50117, epitope: amino acids 2-61 of human Orai1) and rabbit polyclonal anti- $\beta$-actin antibody (catalog number A2066, epitope: amino acids 365-375 of human $\beta$-actin) were obtained from Sigma (St Louis, MO, USA). Horseradish peroxidase-conjugated goat anti-mouse immunoglobulin $\mathrm{G}(\mathrm{IgG})$ antibody and goat anti-rabbit $\operatorname{IgG}$ antibody were from Jackson laboratories (West Grove, PA, USA). CMV-promoter EYFP-Orai1 plasmid (which might give Orai $1 \alpha$ and Orai $1 \beta$ ) was kindly provided by Christoph 
Romanin (Institute of Biophysics, Johannes Kepler University Linz, Austria). CMV-promoter Orai $1 \alpha$-EGFP and Orai $1 \beta$-EGFP plasmids were kindly provided by Mohamed Trebak (Department of Cellular and Molecular Physiology, The Pennsylvania State University, Hershey, PA, USA), been optimized to give essentially $100 \%$ Orai $1 \alpha$ and $100 \%$ Orai $1 \beta$, respectively. Green fluorescent genetically encoded $\mathrm{Ca}^{2+}$ indicator for optical imaging (version 1.2) (G-GECO1.2)Orai1 was a gift from Michael Cahalan (Addgene plasmid \#73562; http://n2t.net/addgene:73562; Research Resource Identifier: Addgene_73562). TRPC1-flag was a gift from Craig Montell (Addgene plasmid \# 24408; http://n2t. net/addgene:24408; RRID:Addgene_24408). pRK5-HATRPC1-F562A (dnTRPC1) plasmid was a gift from Jessica Sabourin (Inserm, UMR-S 1180, Signalisation et Physiopathologie Cardiovasculaire, Université Paris-Saclay, Châtenay-Malabry, France). MO7O-Orai1-E106Q-flag (dnOrai1) was provided by Thierry Capiod (INSERM U1151, Institut Necker Enfants Malades, Université Paris Descartes, Paris, France). MO91-STIM1-CFP was a gift from Anjana Rao (Addgene plasmid \#19755; http://n2t.net/addgene:19755; RRID:Addgene_19755). All other reagents were of an analytical grade.

\section{Site-directed mutagenesis}

Plasmids encoding GECO-Orai1 and Orai $1 \beta$-EGFP were used as template to generate their respective dominantnegative mutants GECO-Orai1-E106Q and Orai1/E43QEGFP (corresponding to the Orai $1 \alpha E 106 Q-E G F P$ mutant), using high-fidelity PCR kit (Platinum ${ }^{\mathrm{TM}}$ SuperFi $^{\mathrm{TM}}$ DNA Polymerase, Invitrogen) and the following primers; ORAI1E106Q_F: 5'-GGTGGCAATGGTGCAGGTGCAGCTGGA3', ORAI1-E106Q_R: 5'-TCCAGCTGCACCTGCACCATT GCCACC- $3^{\prime}$. Sequencing analysis results are depicted in Fig. S1.

\section{Cell culture and transfections}

HeLa cells were obtained from the American Type Culture Collection (CCL-2; Mansassas, VA, USA) and cultured at $37{ }^{\circ} \mathrm{C}$ with a $5 \% \mathrm{CO}_{2}$ in high-glucose DMEM supplemented with $10 \%(\mathrm{v} / \mathrm{v})$ fetal bovine serum and $100 \mathrm{U} / \mathrm{mL}$ penicillin and streptomycin, as described previously [29]. For transient transfections, cells were grown to $60-80 \%$ confluency and transfected with expression plasmids for Orai $1 \alpha$-GFP, Orai $1-\beta$-GFP, dn-Orai $1 \alpha$, dnOrai $1 \beta$ GFP, GECO-Orai1, GECO-dnOrai1 and STIM1-CFP, TRPC1-FLAG and HA-TRPC1-F562A, depending on the experimental conditions, using DharmaFECT kb transfection reagent and were used $48 \mathrm{~h}$ after transfection. For a 4-component overexpression system (STIM1, Orai1 $\alpha$, Orai1 $\beta$, TRPC1), we use a DNA ratio of $4: 1: 1: 2$, and for the 3-component overexpression system (STIM1, GECOOrai $1 \alpha$, TRPC1 or STIM1, Orai $1 \alpha /$ Orai $1 \beta$, TRPC1), we use a DNA ratio of $2: 1: 1$. For Western blotting, immunoprecipitation and biotinylation assays, cells $\left(2 \times 10^{6}\right)$ were plated in 100-mm Petri dish and cultured for $48 \mathrm{~h}$, while, for calcium imaging, determination of $\mathrm{Mn}^{2+}$ entry and confocal determination of G-GECO1.2 fluorescence assays, cells $\left(4 \times 10^{5}\right)$ were seeded in a $35-\mathrm{mm}$ six-well multi-dish.

\section{Immunoprecipitation and Western blotting}

Immunoprecipitation and Western blotting were performed as described previously [30]. Briefly, cells cultured on 100$\mathrm{mm}$ Petri dish $\left(8 \times 10^{6}\right.$ cells $)$ were stimulated with $3 \mu \mathrm{M}$ histamine or with vehicle and subsequently lysed with ice-cold NP-40 buffer $\mathrm{pH} 8$ containing $137 \mathrm{mM}$ of $\mathrm{NaCl}, 20 \mathrm{mM}$ of Tris, $2 \mathrm{mM}$ of EDTA, $10 \%$ glycerol, $1 \%$ nonidet P-40, $1 \mathrm{mM}$ of $\mathrm{Na}_{3} \mathrm{VO}_{4}$, and complete EDTA-free protease inhibitor tablets. Cell lysates $(1 \mathrm{~mL})$ were immuno-precipitated by incubation with $2 \mu \mathrm{g}$ of anti-Orai1, anti-STIM1 or antiTRPC1 antibody and $50 \mu \mathrm{L}$ of protein A-agarose overnight at $4{ }^{\circ} \mathrm{C}$ on a rotary platform. Orai 1 variants expression and detection were analyzed after protein de-glycosilation by treatment of whole-cell lysates with PNGase F according to the protocols provided by the manufacturer. Cell lysates and immuno-precipitates were resolved by $10 \%$ or $12 \%$ SDS-PAGE and separated proteins were electrophoretically transferred onto nitrocellulose membranes for subsequent probing. Blots were incubated overnight with $10 \%$ (w/v) BSA in Tris-buffered saline with $0.1 \%$ Tween 20 (TBST) to block residual protein-binding sites. Immuno-detection of Orai1 variants, $\beta$-actin, STIM1, PMCA and TRPC1 was achieved by incubation for $1 \mathrm{~h}$ with anti-Orail antibody diluted 1:1000 in TBST, $1 \mathrm{~h}$ with anti- $\beta$-actin antibody diluted 1:2000 in TBST, $1 \mathrm{~h}$ with anti-STIM1 diluted 1:500 in TBST, $2 \mathrm{~h}$ with anti-PMCA diluted 1:1000 in TBST or $2 \mathrm{~h}$ with anti-TRPC1 diluted 1:1000 in TSBT. The primary antibody was removed, and blots were washed six times for 5 min each with TBST. To detect the primary antibody, blots were incubated for $1 \mathrm{~h}$ with horseradish peroxidase-conjugated goat anti-mouse IgG antibody, horseradish peroxidaseconjugated goat anti-rabbit IgG antibody diluted 1:10,000 in TBST, or Clean-Blot ${ }^{\mathrm{TM}}$ IP Detection Reagent diluted 1:250 in TBST, and then exposed to enhanced chemiluminescence reagents for $5 \mathrm{~min}$. The antibody binding was assessed with a C-DiGit Chemiluminescent Western Blot Scanner (LICOR Biosciences, Lincoln, NE, USA) and the density of bands was measured using ImageJ software v.1.8.0_172 (NIH, Bethesda, MD, USA). Data were normalized to the amount of protein recovered by the antibody used for the immunoprecipitation or to $\beta$-actin from the same gel. 


\section{APEX2 proximity labeling assay}

The APEX2 proximity labeling assays were performed as described previously [31]. Briefly, biotin-phenol labeling was initiated by cell incubation at $37{ }^{\circ} \mathrm{C}$ with a $5 \% \mathrm{CO}_{2}$ for $30 \mathrm{~min}$ in cell culture medium supplemented with $2.5 \mathrm{mM}$ biotin-phenol. Cells were stimulated with $3 \mu \mathrm{M}$ histamine for $5 \mathrm{~min}$ or with vehicle, then medium was removed and cells were washed three time with PBS $\left(10 \mathrm{mM} \mathrm{Na}_{2} \mathrm{HPO}_{4}\right.$, $1.8 \mathrm{mM} \mathrm{KH}_{2} \mathrm{PO}_{4}, 2.7 \mathrm{mM} \mathrm{KCL}, 140 \mathrm{mM} \mathrm{NaCL}, 0.5 \mathrm{mM}$ $\mathrm{MgCl}_{2}, 1 \mathrm{mM} \mathrm{CaCl}$, $\mathrm{pH}$ 7.4) and incubated for $1 \mathrm{~min}$ in biotinylation buffer (PBS supplemented with $1 \mathrm{mM} \mathrm{H}_{2} \mathrm{O}_{2}$ ). Later, biotinylation buffer was aspired and cells were washed three times with STOP/wash buffer (PBS containing: $0.5 \mathrm{mM} \mathrm{MgCl}_{2}, 1 \mathrm{mM} \mathrm{CaCl}_{2}, 5 \mathrm{mM}$ Trolox, $10 \mathrm{mM}$ sodium ascorbate, $10 \mathrm{mM}$ sodium azide). Cells were subsequently lysed with ice-cold lysis (RIPA) buffer $\mathrm{pH} 8(50 \mathrm{mM}$ Tris, $150 \mathrm{mM} \mathrm{NaCl}, 5 \mathrm{mM}$ EDTA, $0.5 \%$ sodium deoxycholate, 0,1\% SDS, $1 \%$ Triton X-100, complete EDTA-free protease inhibitor cocktail) supplemented with $5 \mathrm{mM}$ Trolox, $10 \mathrm{mM}$ sodium ascorbate, $10 \mathrm{mM}$ sodium azide and then cells were scraped. Cell lysates were transferred to micro-centrifuge tubes and samples were sonicated. Clear cell lysates were obtained by centrifugation at $20,000 \times g$ for $30 \mathrm{~min}$ at $4{ }^{\circ} \mathrm{C}$ and total protein concentrations were determined using the BCA method. Biotinylated proteins were isolated by incubation with $50 \mu \mathrm{L}$ of streptavidin beads at $4{ }^{\circ} \mathrm{C}$ for $2 \mathrm{~h}$ on a rotary platform. Later, beads were washed twice with $1 \mathrm{~mL}$ RIPA buffer, once with $1 \mathrm{~mL} 1 \mathrm{M} \mathrm{KCl}$, once with $1 \mathrm{~mL}$ $0.1 \mathrm{M} \mathrm{Na}_{2} \mathrm{CO}_{3}$, once with $1 \mathrm{~mL} 2 \mathrm{M}$ urea in Tris- $\mathrm{HCl} \mathrm{pH}$ 8.0 and once with $1 \mathrm{~mL}$ RIPA buffer. For Western blotting assay, the biotinylated and non-biotinylated fractions were then eluted by boiling the beads at $95{ }^{\circ} \mathrm{C}$ for $15 \mathrm{~min}$ in Laemmli buffer (0.62 M Tris-Cl pH 6.8, 2\% SDS, $10 \%$ glycerol, $0.002 \%$ bromophenol blue) supplemented with $100 \mathrm{mM}$ DTT and $1 \mathrm{mM}$ biotin, and Western blotting was performed as described above.

\section{Determination of cytosolic free $\mathrm{Ca}^{2+}$ concentration}

Cells were loaded with fura- 2 by incubation with $5 \mu \mathrm{M}$ fura-2/AM for $30 \mathrm{~min}$ at $37{ }^{\circ} \mathrm{C}$. Coverslips with cultured cells were mounted on a perfusion chamber and placed on the stage of an epifluorescence inverted microscope (Nikon Eclipse Ti2, Amsterdam, The Netherlands) with an image acquisition and analysis system for video-microscopy (NISElements Imaging Software v.5.02.00, Nikon, Amsterdam, The Netherlands). Cells were continuously super-fused at room temperature with HEPES-buffered saline (HBS) containing (in $\mathrm{mM}$ ) $125 \mathrm{NaCl}, 5 \mathrm{KCl}, 1 \mathrm{MgCl}_{2}, 5$ glucose, and 25 HEPES, pH 7.4, supplemented with $0.1 \%(w / v)$ BSA. Cells were examined at $40 \times$ magnification (Nikon CFI S FLUOR 40× Oil, Amsterdam, The Netherlands) and were alternatively excited with light from a xenon lamp passed through a high-speed monochromator Optoscan ELE 450 (Cairn Research, Faversham, UK) at 340/380 nm. Fluorescence emission at $505 \mathrm{~nm}$ was detected using a cooled digital sCMOS camera Zyla 4.2 (Andor, Belfast, UK) and recorded using NIS-Elements AR software (Nikon, Amsterdam, The Netherlands). Fluorescence ratio (F340/F380) was calculated pixel by pixel, and the data were presented as $\Delta \mathrm{F}_{340} / \mathrm{F}_{380}$. Histamine-evoked changes in cytosolic free $\mathrm{Ca}^{2+}$ concentration were estimated as the area under the curve measured as the integral of the rise in fura-2 fluorescence ratio 10 min after the addition of histamine and taking a sample every second. TG-induced $\mathrm{Ca}^{2+}$ entry was estimated as the integral of the rise in fura-2 fluorescence ratio $2.5 \mathrm{~min}$ after the addition of $\mathrm{Ca}^{2+}$ and taking a sample every second.

\section{Analysis of $\mathrm{Ca}^{2+}$ oscillations}

The analysis of $\mathrm{Ca}^{2+}$ oscillations was performed as described previously [13]. All $\mathrm{Ca}^{2+}$ traces obtained in the $\mathrm{Ca}^{2+}$-imaging experiments were plotted using GraphPad Prism v.8.4.3 (GraphPad Software, San Diego, CA, USA). The numbers of oscillations per 10 min were manually counted. Then, cells were classified in three groups and a percentage of each group of cells was calculated for each individual coverslip. The first group, oscillating cells, includes cells showing regenerative oscillations after histamine stimulation for the duration of the experiment, where each oscillation returns to baseline before the start of the next oscillation. A second group, plateau cells, includes cells showing a sustained a cytosolic $\mathrm{Ca}^{2+}$ signal that was $\geq 25 \%$ of the initial peak, for at least 5 min after stimulation. Finally, the last group includes cells that show either no response to histamine stimulation or showed only one initial spike and remained at baseline for the duration of the recording. All manual counts were independently recorded by two individuals to ensure accuracy of counts.

\section{Determination of $\mathrm{Mn}^{2+}$ entry}

Cells were loaded with fura- 2 by incubation with $5 \mu \mathrm{M}$ fura-2/AM for $30 \mathrm{~min}$ at $37{ }^{\circ} \mathrm{C}$. Coverslips with cultured cells were mounted on a perfusion chamber and placed on the stage of an epifluorescence inverted microscope (Nikon Eclipse Ti2, Amsterdam, The Netherlands) with an image acquisition and analysis system for video-microscopy (NISElements Imaging Software v.5.02.00, Nikon, Amsterdam, The Netherlands). Cells were continuously super-fused at room temperature with HEPES-buffered saline (HBS) containing (in mM) $1 \mathrm{CaCl}_{2}, 0.5 \mathrm{MnCl}_{2}, 125 \mathrm{NaCl}, 5 \mathrm{KCl}, 1$ $\mathrm{MgCl}_{2}, 5$ glucose, and 25 HEPES, $\mathrm{pH}$ 7.4, supplemented with $0.1 \%(w / v)$ BSA. Cells were examined at $40 \times$ magnification (Nikon CFI S FLUOR 40× Oil, Amsterdam, The 
Netherlands) and were excited with light from a xenon lamp passed through a high-speed monochromator Optoscan ELE 450 (Cairn Research, Faversham, UK) at $360 \mathrm{~nm}$. Fluorescence emission at $505 \mathrm{~nm}$ was detected using a cooled digital sCMOS camera Zyla 4.2 (Andor, Belfast, UK) and recorded using NIS-Elements AR software (Nikon, Amsterdam, The Netherlands). $\mathrm{Mn}^{2+}$ influx was monitored as the quenching of fura-2 fluorescence at the isoemissive wavelength of $360 \mathrm{~nm}$ and presented on an arbitrary linear scale. To compare the rate of decay of fura- 2 fluorescence when cells were subjected to different experimental procedures, traces were fitted to the equation $y=S \times e^{-K X}+A$, where $K$ is the slope, $S$ is the span and $A$ is the plateau, as described previously [32].

\section{Confocal determination of G-GEC01.2 fluorescence}

\section{G-GECO1.2-Orai1 or G-GECO1.2-dnOrai1-transfected} Hela cells were seeded on coverslips and mounted on a perfusion chamber and placed on the stage of an epifluorescence inverted microscope (Nikon Eclipse Ti, Amsterdam, The Netherlands) with an image acquisition and analysis system for video-microscopy NIS-Elements Imaging Software v.5.02.00, (Nikon, Amsterdam, The Netherlands). Cells were continuously super-fused with HBS supplemented with $0.1 \%(w / v)$ BSA at room temperature. Cells were examined at $60 \times$ magnification and excited using a confocal laserscanning system (Melles-Griot, IDEX Health \& Science, Wallingford, CT, USA) at $488 \mathrm{~nm}$. Fluorescence emission at $515 \mathrm{~nm}$ was detected and recorded using NIS-Elements AR software (Nikon, Amsterdam, The Netherlands). GECO fluorescence was determined before and after the addition of $3 \mu \mathrm{M}$ histamine (resting) in presence of extracellular $\mathrm{Ca}^{2+}$ $(1 \mathrm{mM})$ for $10 \mathrm{~min}$. Images were analyzed using ImageJ software v.1.8.0_172 (NIH, Bethesda, MD, USA).

\section{Biotinylation of cell surface proteins}

The labeling and isolation of cell surface proteins were performed by surface biotinylation assay, as described previously [33]. Hela cells grown in $75 \mathrm{~cm}^{2}$ tissue culture dishes were washed three times with phosphate-buffered saline (PBS, $\mathrm{NaCl} 137 \mathrm{mM}, \mathrm{KCl} 2.7 \mathrm{mM}, \mathrm{KH}_{2} \mathrm{PO} 4,1.5 \mathrm{mM}$, $\mathrm{Na}_{2} \mathrm{HPO}_{4} \cdot 2 \mathrm{H}_{2} \mathrm{O} 8 \mathrm{mM}, \mathrm{pH}$ 8). Later, cells were incubated at $4{ }^{\circ} \mathrm{C}$ for $1 \mathrm{~h}$ with biotynilation buffer (PBS supplemented with $1 \mathrm{mg} / \mathrm{mL}$ EZ-Link sulfo-NHS-LC-biotin) The biotinylation reaction was terminated by addition of Tris base to a final concentration of $50 \mathrm{mM}$. Following biotinylation, cells were washed twice in PBS, disrupted using Nonidet P-40 buffer and sonicated. Cell lysates were centrifuged $\left(16,000 \times g\right.$ for 5 min at $\left.4{ }^{\circ} \mathrm{C}\right)$ and protein concentration was measured using BCA assay. Samples were incubated with $50 \mu \mathrm{L}$ streptavidin beads at $4{ }^{\circ} \mathrm{C}$ for $2 \mathrm{~h}$ and re-suspended in Laemmli buffer for subsequent analysis by Western blotting. The biotinylated and non-biotinylated fractions were separated in $8 \%$ SDS-PAGE, TRPC1 surface expression was detected using a specific anti-TRPC1 antibody, while the detection of PMCA was used as control.

\section{Statistical analysis}

All data are presented as the mean \pm standard error of mean (SEM). Analysis of statistical significance was performed using GraphPad Prism v.8.4.3 (GraphPad Software, San Diego, CA, USA). Kruskal-Wallis test combined with Dunn's post hoc test (or one-way analysis of variance combined with Tukey post hoc test for the analysis of $\mathrm{Ca}^{2+}$ determinations) was used to compare the different experimental groups. For comparison between two groups, the Mann-Whitney U test was used. Throughout the manuscript $*, * *$, and $* * *$ indicate $p$ values of $<0.05,<0.01$, and $<0.001$, respectively. All data with $p<0.05$ were deemed significant; "ns" = nonsignificant.

\section{Results}

\section{Orai1 $a$ and Orai1 $\beta$, but not TRPC1, are important to modulate $\mathrm{Ca}^{2+}$ oscillations}

We have analyzed the functional role of Orai $1 \alpha$, Orai $1 \beta$ and TRPC1 on histamine-induced $\mathrm{Ca}^{2+}$ oscillations. HeLa cells were co-transfected with STIM1-CFP, Orai1 $\alpha$ EGFP, Orai $1 \beta$-EGFP and TRPC1 or the corresponding dominant-negative mutants for Orai $1 \alpha$ (Orai1 $\alpha$ E106Q), Orai1 $\beta$ (Orai1 $\beta E 43 Q-E G F P$ corresponding to the E106Q mutant of the long Orai1 variant) and TRPC1 (HA-TRPC1F562A). Expression of the different plasmids was demonstrated by Western blotting using the appropriate antibodies (Fig. S2). Cells were stimulated with $3 \mu \mathrm{M}$ histamine to elicit $\mathrm{Ca}^{2+}$ oscillations in the presence of $1 \mathrm{mM}$ extracellular $\mathrm{Ca}^{2+}$. Traces from five representative cells are shown in Fig. 1a-h. An average of $29 \%$ of HeLa cells expressing WT STIM1, Orai $1 \alpha$, Orai $1 \beta$ and TRPC1 stimulated with histamine responded with regenerative $\mathrm{Ca}^{2+}$ oscillations (Fig. 1i), with an average of $5.7 \pm 0.2$ oscillations $/ 10 \mathrm{~min}$ (Fig. 11). Of the cells that did not oscillate, $68 \%$ responded with a sustained plateau and the remaining $3 \%$ of cells did not respond (Fig. 1j, k). In mock-treated cells, an average of about $20 \%$ responded with $\mathrm{Ca}^{2+}$ oscillations after stimulation with histamine, with an average of 4 oscillations/10 min (Fig. S3a-c). As expected, the magnitude of $\mathrm{Ca}^{2+}$ mobilization upon treatment with histamine was significantly greater in cells expressing STIM1, Orai $1 \alpha$, Orai $1 \beta$ and TRPC1 (Fig. $\mathrm{S} 3 \mathrm{~d} ; p<0.001)$. 


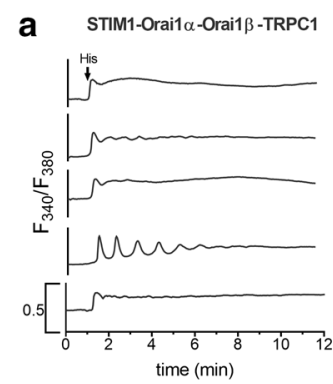

e STIM1-Orai1 $\alpha$-Orai1 $\beta$-dnTRPC1
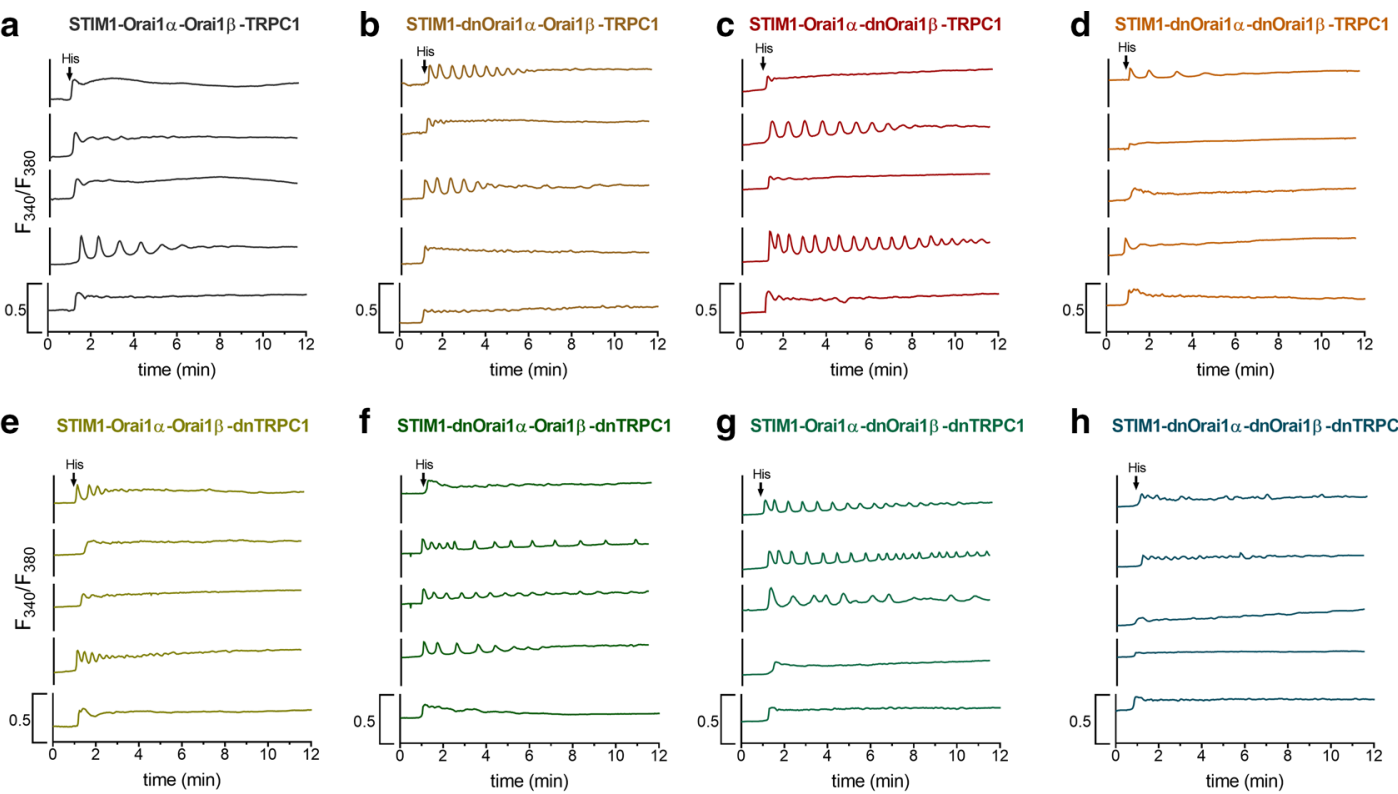

g STIM1-Orai1 $\alpha$-dnOrai1 $\beta-$ dnTRPC1

h STIM1-dnOrai1 $\alpha$-dnOrai1 $\beta$-dnTRPC1
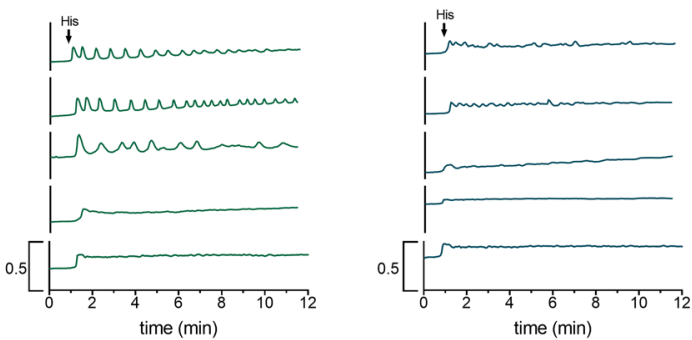

j
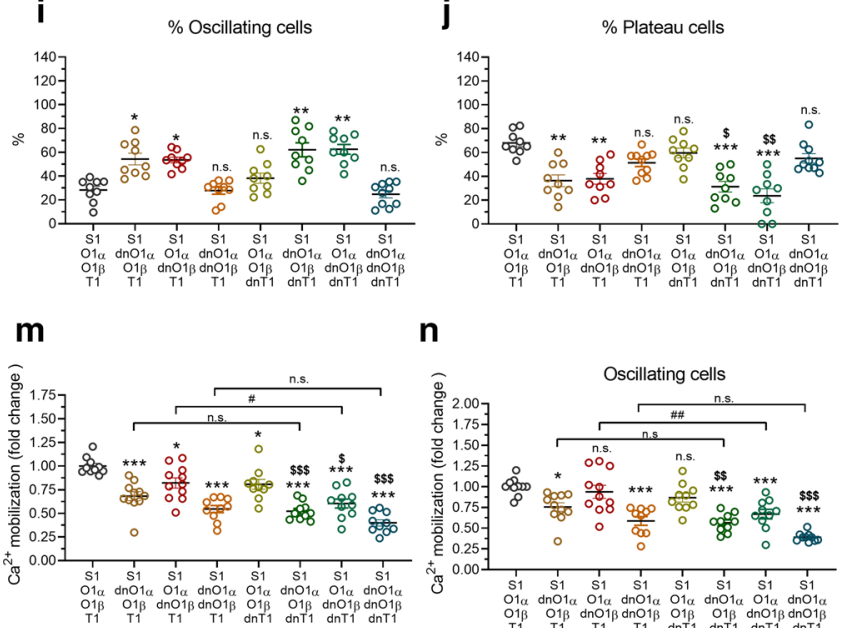

k

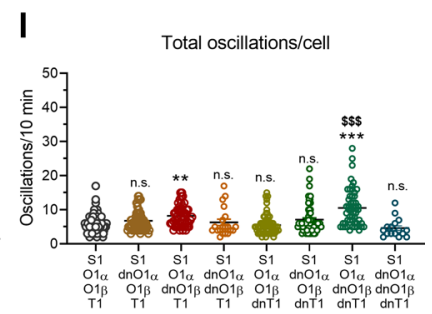

o

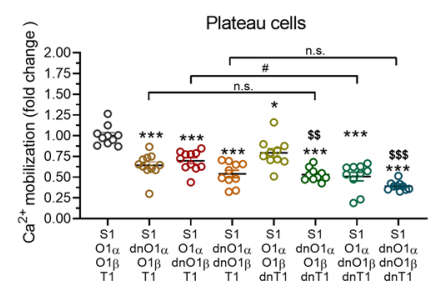

Fig. 1 Orai $1 \alpha$ and Orai $1 \beta$, but not TRPC1, are important to modulate $\mathrm{Ca}^{2+}$ oscillations. a-h Representative $\mathrm{Ca}^{2+}$ oscillations in response to $3 \mu \mathrm{M}$ histamine measured using fura- 2 in HeLa cells co-transfected with STIM1, Orai1 $\alpha$, Orai1 $\beta$ and TRPC1 or the corresponding dominant-negative mutants, as described. Cells were super-fused with HBSS containing $1 \mathrm{mM} \mathrm{Ca}^{2+}$ and stimulated with $3 \mu \mathrm{M}$ histamine at $1 \mathrm{~min}$ (indicated by arrow). Representative traces from five cells/condition were chosen to represent the datasets. $\mathbf{i}-\mathbf{l}$ Quantification of the percentage of oscillating cells (i), percentage of plateau cells $(\mathbf{j})$, percentage of non-responding cells $(\mathbf{k})$ and total oscillations/cell in $10 \min$ (I) for data presented in $\mathbf{a}-\mathbf{h}$ (for $\mathbf{i}$ to $\mathbf{k}, n=9$; $n$ values correspond to independent experiments; for $\mathbf{l}$, from left to

When Orai $1 \alpha$ or Orai $1 \beta$ were substituted by Orai $1 \alpha$ E106Q (dnOrai $1 \alpha)$ or Orai1 $\beta$-E43Q (dnOrai1 $\beta)$ mutants, the percentage of cells that responded with $\mathrm{Ca}^{2+}$ oscillations significantly increased (57\% and 56\%, for Orai $1 \alpha$ and Orai $1 \beta$, respectively; $p<0.05$; Fig. 1b, c, i). Interestingly, expression of the dnOrai $1 \beta$ mutant significantly enhanced the average number of oscillations per cell $(8.2 \pm 0.4$ oscillations $/ 10 \mathrm{~min}$; right, $n=99,68,46,20,73,62,53$ and $16 ; \mathrm{n}$ values correspond to individual cells). $\mathbf{m}-\mathbf{0}$ Quantification of $\mathrm{Ca}^{2+}$ mobilization for all the conditions from a to $\mathrm{h}$ estimated in all the cells (m), oscillating cells (n) and plateau cells (o). Scatter plots are represented as mean \pm SEM and were statistically analyzed using Kruskal-Wallis test with multiple comparisons (Dunn's test) to HeLa cells expressing WT Orai $\alpha$, Orai $1 \beta$ and TRPC1 $(* p<0.05, * * p<0.01$, and $* * * p<0.001)$, HeLa cells expressing WT Orai $1 \alpha$, WT Orai $1 \beta$ and dnTRPC1 (for conditions including the expression of dnTRPC1; ${ }^{\$} p<0.05,{ }^{\$}{ }_{p}<0.01$, and $\left.{ }^{\$ \$} p<0.001\right)$ or the corresponding condition with WT TRPC1 vs dnTRPC1 $\left({ }^{\#} p<0.05,{ }^{\# \#} p<0.01\right.$, and $\left.{ }^{\# \# \#} p<0.001\right)$.

Fig. 11). However, co-transfection of HeLa cells with STIM1, TRPC1 and both dominant-negative mutants of Orai $1 \alpha$ and Orai $1 \beta$ returned the percentage of cells that responded with $\mathrm{Ca}^{2+}$ oscillations to that observed in cells expressing WT STIM1, Orai $1 \alpha$, Orai1 $\beta$ and TRPC1 (Fig. 1d, i).

To ascertain the possible role TRPC 1 in the maintenance or $\mathrm{Ca}^{2+}$ oscillations, we repeated the previously described 
protocols in the presence of the dominant-negative TRPC1 mutant (HA-TRPC1-F562A; dnTRPC1). As shown in Fig. 1e-1, substitution of WT TRPC1 for dnTRPC1 did not significantly alter the response pattern of the cells to histamine. Thus, suggesting that TRPC1 does not play a relevant role in the maintenance of regenerative $\mathrm{Ca}^{2+}$ oscillations in these cells.

Concerning the magnitude of histamine-evoked $\mathrm{Ca}^{2+}$ mobilization, co-transfection of dnOrai $1 \alpha$ or dnOrai $1 \beta$, instead of the WT Orai1 variants, with STIM1 and TRPC1 led to a significant attenuation of histamine-evoked $\mathrm{Ca}^{2+}$ mobilization (Fig. 1m; $p<0.05$ ), which affected exclusively to the cells that responded with a sustained plateau (Fig. 1n, o). Consistent with this, substitution of both WT Orai1 variants for their respective dominant-negative mutants further decreased $\mathrm{Ca}^{2+}$ mobilization induced by histamine, which, in this case, affected to both oscillating cells and cells that responded with a sustained plateau (Fig. $1 \mathrm{~m}-\mathrm{o} ; p<0.001$ ). Co-transfection of dnTRPC1 with STIM1, Orai1 $\alpha$ and Orai $1 \beta$ (or the corresponding dnOrai $1 \alpha$ and dnOrai $1 \beta$ ) significantly decreased histamine-evoked $\mathrm{Ca}^{2+}$ mobilization as compared to HeLa cells expressing WT TRPC1 (Fig. 1m-O; $p<0.05$ to 0.001$)$. In the presence of dnTRPC 1 , agonistinduced $\mathrm{Ca}^{2+}$ mobilization was still smaller when $\mathrm{dnOrai} 1 \alpha$, dnOrai $1 \beta$ or both were expressed (Fig. 1n, o). These findings indicate that, while Orai $1 \alpha$, Orai $1 \beta$ and TRPC 1 are required for histamine-induced $\mathrm{Ca}^{2+}$ mobilization, only Orai $\alpha$ and Orai $1 \beta$ play a relevant role in the maintenance of regenerative $\mathrm{Ca}^{2+}$ oscillations.

We have further analyzed the functional role of Orai $1 \alpha$, Orai $1 \beta$ and TRPC 1 on histamine-induced $\mathrm{Ca}^{2+}$ oscillations using triple co-expression of STIM1, Orai $1 \alpha$ or Orai $1 \beta$ and TRPC1, or the corresponding dominant-negative mutants. Traces from five representative cells are shown in Fig. $\mathrm{S} 4 \mathrm{a}-\mathrm{h}$. With this experimental maneuver, an average of $51 \pm 3$ and $52 \pm 5 \%$ of HeLa cells expressing WT STIM1, Orai $1 \alpha$ or Orai $1 \beta$, respectively, and TRPC 1 stimulated with histamine responded with regenerative $\mathrm{Ca}^{2+}$ oscillations (Fig. S4i), with an average of $9.2 \pm 0.7$ and $7.6 \pm 0.7$ oscillations/10 min, for Orail $\alpha$ and Orai $1 \beta$ (Fig. S4l). When the WT Orai1 variants were replaced by their corresponding dominant-negative mutants, the percentage of cells that responded with $\mathrm{Ca}^{2+}$ oscillations significantly decreased to $18 \pm 3 \%$ and $21 \pm 2 \%$, for Orai $\alpha$ and Orai $1 \beta$, respectively, and the average number of oscillations per cell was attenuated $(3.8 \pm 0.6$ and $4.6 \pm 0.5$ oscillations $/ 10 \mathrm{~min}$, for Orai $1 \alpha$ and Orai $1 \beta$, respectively; Fig. S4c, d, i, $1 ; p<0.05)$. As expected, substitution of the Orail variants by their respective dominant-negative mutants significantly attenuated the magnitude of histamine-evoked $\mathrm{Ca}^{2+}$ mobilization (Fig. $\mathrm{S} 4 \mathrm{~m}-\mathrm{o} ; p<0.05)$. These findings confirm that both variants of Orail are required for agonist-induce $\mathrm{Ca}^{2+}$ oscillations. We have further evaluated the role of TRPC1 in this triple co-expression system replacing TRPC1 by dnTRPC1. Substitution of WT TRPC1 for dnTRPC1 did not significantly alter the response pattern of the cells to histamine as reported in Fig. 1, thus suggesting that TRPC1 does not play a relevant role in the maintenance of regenerative $\mathrm{Ca}^{2+}$ oscillations in these cells. Interestingly, expression of dnTRPC1 instead of WT TRPC1 significantly attenuated the magnitude of agonist-evoked $\mathrm{Ca}^{2+}$ mobilization exclusively in cells expressing functional Orail $\alpha$ (Fig. S4m; $p<0.01$ ).

\section{Analysis of the interaction of Orai1 $\alpha$ and Orai1 $\beta$ with TRPC1}

We have explored the interaction between the Orail variants, Orai $1 \alpha$ and Orai $1 \beta$, and TRPC1 in native HeLa cells by looking for co-immunoprecipitation from cell lysates. Immunoprecipitation and subsequent SDS/PAGE and Western blotting were conducted using resting cells and cells in which the intracellular $\mathrm{Ca}^{2+}$ stores had been depleted by 1 min treatment with thapsigargin (TG; $2 \mu \mathrm{M}$ ) in the presence of extracellular $\mathrm{Ca}^{2+}(1 \mathrm{mM})$. After immunoprecipitation with the anti-TRPC1 antibody, and protein de-glycosylation with PNGaseF, Western blotting revealed the presence of Orai $1 \alpha$ and, in a significantly less amount, Orai1 $\beta$ in samples from resting cells. (Fig. 2a, top panel). Treatment with TG significantly increased the amount of Orai $1 \alpha$ immunoprecipitated with anti-TRPC1 antibody (Fig. 2a, top panel, and $c ; p<0.05)$ without having any effect on the interaction between Orai1 $\beta$ and TRPC1 (Fig. 2a, c). Western blotting with anti-TRPC1 antibody confirmed a similar amount of protein in all lanes (Fig. 2a, bottom panel).

Previous studies have demonstrated functional interaction of both Orai1 variants with STIM1 [4, 15]. As a positive control of our experimental procedure, we have evaluated the interaction of STIM1 with Orai $1 \alpha$ and Orai $1 \beta$ following the previously described experimental maneuver. As shown in Fig. 2b, top panel, after immunoprecipitation with the anti-STIM1 antibody and protein de-glycosylation with PNGaseF, Western blotting reveals a low amount of Orai1 variants associated with STIM1 in resting cells. The association of Orai $1 \alpha$ and Orai $1 \beta$ with STIM 1 significantly increased after treatment with TG (Fig. 2b, d; $p<0.05$ ). Western blotting with anti-STIM1 antibody confirmed a similar amount of protein in all lanes (Fig. 2b, bottom panel).

We also analyzed the interaction between TRPC1 and Orail variants by immunoprecipitation using an alternative experimental maneuver. As shown in Fig. S5, HeLa cells were suspended in a $\mathrm{Ca}^{2+}$-free medium $(300 \mu \mathrm{M}$ EGTA added) or a medium containing $1 \mathrm{mM} \mathrm{CaCl}_{2}$ and were stimulated with TG or left untreated and lysed. Orail $\alpha$ was pulled down by immunoprecipitation of the whole-cell lysates with anti-Orai1 N-terminal antibody (epitope: amino acids 2-61 exclusive to Orai $1 \alpha$ ). The immuno-precipitates 


\section{a}
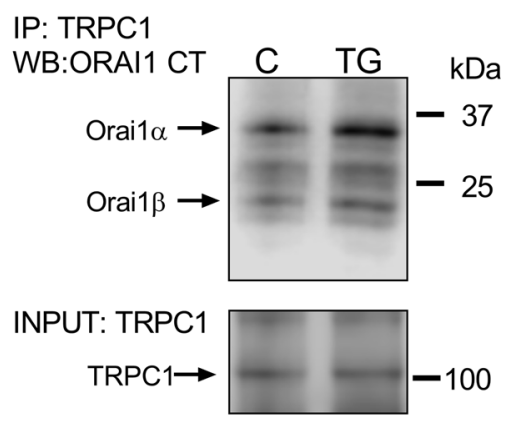

C

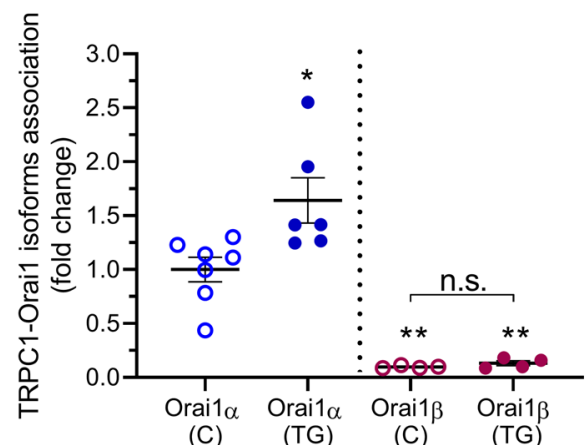

e

\section{BIOTINYLATED FRACTION}

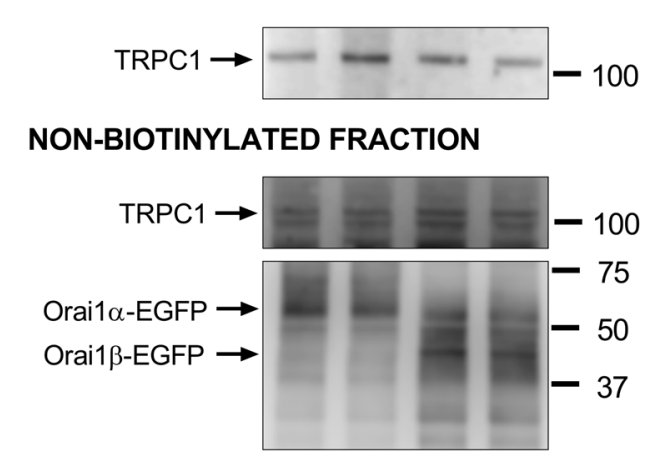

b
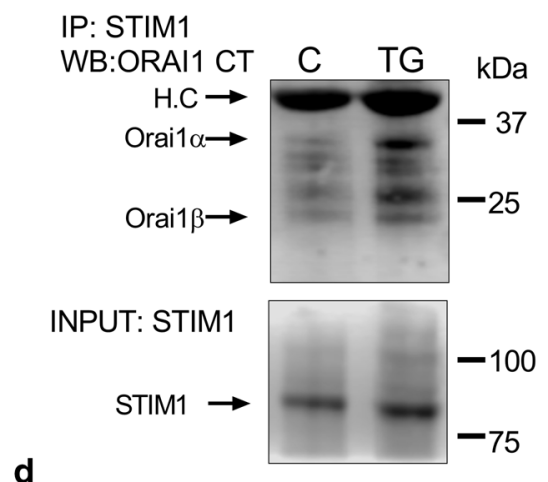

d

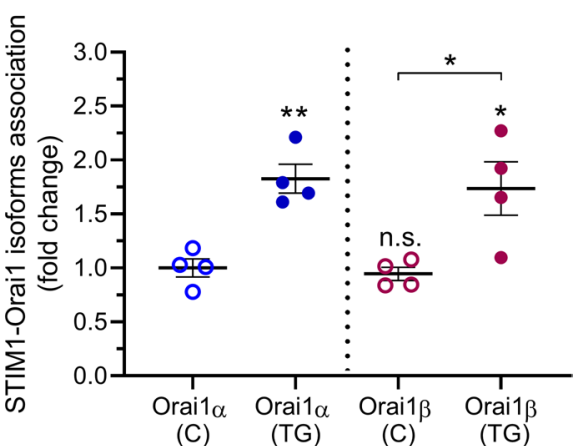

f

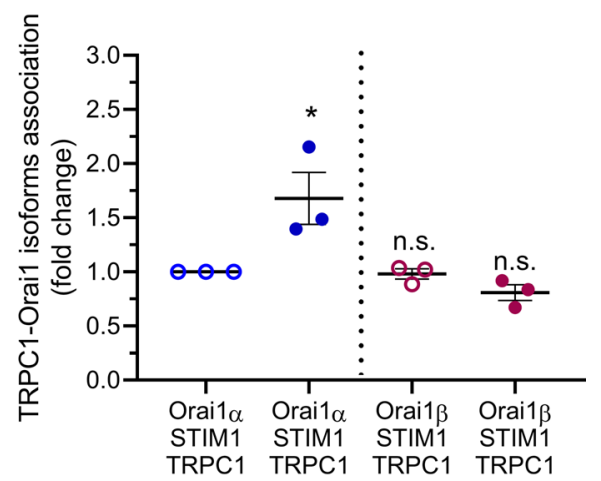

(C)

(C)

(H)
Fig. 2 Interaction of Orai1 $\alpha$ and Orai1 $\beta$ with TRPC1 and STIM1. HeLa cells were suspended in HBS containing $1 \mathrm{mM} \mathrm{Ca}^{2+}$ and then stimulated for 1 min with $2 \mu \mathrm{M}$ TG (TG) or the vehicle (C) and lysed. Whole-cell lysates were immuno-precipitated with anti-TRPC1 (a) or anti-STIM1 antibody (b). Immuno-precipitates were treated with PNGaseF and then subjected to $10 \%$ SDS-PAGE and Western blotting with the anti-Orail antibody, as described in Material and Methods. Membranes were re-probed with the antibody used for immunoprecipitation for protein loading control. Molecular masses indicated on the right were determined using molecular-mass markers run in the same gel. Blots are representative of four to seven separate experiments. c, d Scatter plots, representing the quantification of the TRPC1-Orai1 $\alpha / \beta$ or STIM1-Orai1 $\alpha / \beta$ interaction, are presented as mean \pm SEM and were statistically analyzed using the Mann-Whitney $\mathrm{U}$ test $\left(* p<0.05\right.$ and ${ }^{*} p<<0.01$, as compared to the corresponding control (C)). e HeLa cells were co-transfected with STIM1-CFP, TRPC 1 and either Orai1 $\alpha$-GFP (lanes 1 and 2) or Orai1 $\beta$-GFP (lanes
3 and 4). Cells were suspended in HBS containing $1 \mathrm{mM} \mathrm{Ca}^{2+}$ and then stimulated with $3 \mu \mathrm{M}$ histamine (lanes 2 and 4 ) or the vehicle (lanes 1 and 3) for $5 \mathrm{~min}$, and the interaction between Orai1 variants and TRPC1 was assessed by APEX 2 proximity labeling assay, as described in Material and Methods. The biotinylated and nonbiotinylated fractions were subjected to $10 \%$ SDS-PAGE and Western blotting with the anti-TRPC1 antibody. The non-biotinylated fraction was also probed with the anti-Orailantibody for protein loading control. Molecular masses indicated on the right were determined using molecular-mass markers run in the same gel. Blots are representative of three separate experiments. f Scatter plots, representing the quantification of the TRPC1-Orai1 $\alpha$ or TRPC1-Orai1 $\beta$ interaction in resting $(\mathrm{C}$; control) and histamine $(\mathrm{H})$-treated cells, are presented as mean \pm SEM and were statistically analyzed using the Mann-Whitney $U$ test $\left({ }^{*} p<0.05\right.$, as compared to the corresponding control). $H C$ heavy chain of the IgG used for immunoprecipitation 
were subjected to SDS/PAGE and Western blotting with anti-TRPC1 antibody to detect the Orai1 $\alpha$-TRPC1 association. On the other hand, Orai $1 \beta$ was pulled down from the supernatant by immunoprecipitation with anti-Orai1 (C-terminal) antibody (epitope: amino acids 288-301 present in both Orail variants). The resulting pellet was subjected to SDS/PAGE and Western blotting with anti-TRPC1 antibody to detect the Orai1 $\beta$-TRPC1 association. A detectable association was found between Orai1 $\alpha$ and TRPC 1 both in the absence and presence of extracellular $\mathrm{Ca}^{2+}$ but we were unable to detect a significant interaction between TRPC1 and Orai $1 \beta$ (Fig. S5).

We have further tested the interaction between Orai1 variants and TRPC 1 by proximity-dependent biotinylation using APEX2. HeLa cells were co-transfected with STIM1-CFP, TRPC1 and either Orai1 $\alpha$-EGFP or Orai $1 \beta$-EGFP. Experiments were performed in resting cells and cells stimulated with $3 \mu \mathrm{M}$ histamine for $5 \mathrm{~min}$ in the presence of extracellular $\mathrm{Ca}^{2+}(1 \mathrm{mM})$. As shown in Fig. 2e, top panel, the resulting biotinylation pattern reveals a small but detectable interaction of TRPC 1 with Orai $1 \alpha$ and Orai $1 \beta$ in resting conditions. Interestingly, treatment with histamine significantly enhances the Orai $1 \alpha-$ TRPC 1 interaction without inducing any modification in the interaction between TRPC1 and Orai1 $\beta$ (Fig. 2e, top panel, and f). Western blot analysis of the non-biotinylated fraction with anti-TRPC1 antibody revealed the amount of TRPC1 not associated to Orai1 variants (Fig. 1e, middle panel). Further, re-probing of the nonbiotinylated fraction with anti-Orai1 (C-terminal) antibody confirmed a similar amount of Orail variants in all lanes (Fig. 2e, bottom panel).

Altogether, either using co-immunoprecipitation or APEX2 proximity labeling assay, our results indicate that there is a detectable interaction of TRPC 1 with Orai $1 \alpha$ under resting conditions that was significantly enhanced by treatment with TG or physiological agonists. By contrast, there is a small, if any, interaction between Orai $1 \beta$ and TRPC1 that seems constitutive in nature.

Alternatively, we have analyzed the proximity between TRPC 1 and Orai $1 \alpha$ by looking for the influence of $\mathrm{Ca}^{2+}$ influx through TRPC1 on the fluorescence of G-GECO1.2 fused to Orai $1 \alpha$ (G-GECO-Orai1 $\alpha)$. G-GECO-Orai $1 \alpha$ was transiently co-transfected with STIM1 and TRPC1 or dnTRPC1 mutant into HeLa cells and imaged using an epifluorescence inverted microscope. We have previously reported that G-GECO-Orai $1 \alpha$ exhibited diffuse expression in the plasma membrane [30]. Cells were stimulated with $3 \mu \mathrm{M}$ histamine to elicit $\mathrm{Ca}^{2+}$ influx (detected as fluctuations) through Orai $1 \alpha$ in the presence of $1 \mathrm{mM}$ extracellular $\mathrm{Ca}^{2+}$. Traces from five representative cells are shown in Fig. 3a-d. An average of $87 \%$ of HeLa cells expressing STIM1, G-GECO-Orai1 $\alpha$ and TRPC1 stimulated with histamine responded with G-GECO1.2 fluorescence fluctuations
(Fig. 3e), with an average of $11.7 \pm 1.1$ fluctuations/10 min (Fig. 3h). Of the cells that did not fluctuate, $11 \%$ responded with a sustained plateau and the remaining $2 \%$ of cells did not respond (Fig. 3f, g). Substitution of TRPC1 by dnTRPC1 significantly decreased the percentage of fluctuating cells to $58 \%$, while the number of G-GECO1.2 fluorescence fluctuations per cell was unaffected (Fig. 3e-h).

In cells expressing TRPC1, the overall $\mathrm{Ca}^{2+}$ influx detected by G-GECO1.2 was significantly greater than that observed in cells expressing dnTRPC mutant (Fig. 3i-k; $p<0.05)$. These findings suggest that either TRPC1 enhances $\mathrm{Ca}^{2+}$ influx through Orail $\alpha$ or that both channels are very close so that $\mathrm{Ca}^{2+}$ entry through TRPC1 might be detected by Orai1 $\alpha$-fused G-GECO1.2. Concerning the first hypothesis, it is well established that Orai1/STIM1 forms the primary SOCE channel and leads to the initial $\mathrm{Ca}^{2+}$ influx upon depletion of the intracellular $\mathrm{Ca}^{2+}$ stores, while TRPC1, can be recruited to ER-PM junctions and inserted into the PM by a mechanism that requires Orai1-mediated $\mathrm{Ca}^{2+}$ entry [1]. According to this, it is quite unlikely that TRPC1 modulates the onset of $\mathrm{Ca}^{2+}$ influx via Orai1 $\alpha$, but once cation entry through TRPC1 is initiated, there are several mechanisms that might negatively influence Orai $1 \alpha$ function, including changes in the driving force for $\mathrm{Ca}^{2+}$ entry by membrane depolarization, competition for STIM1 units or the facilitation of $\mathrm{Ca}^{2+}$-dependent Orai1 $\alpha$ inactivation [34]. Accordingly, we have investigated whether TRPC1 influences the phosphorylation of Orai $1 \alpha$ at serine residues, which has been demonstrated to induce Orail channel inactivation $[4,35]$. To investigate this phenomenon, HeLa cells were co-transfected with STIM1, Orai $1 \alpha$ and TRPC1 or the dnTRPC1 mutant. Cells were subsequently stimulated with histamine in the presence of $1 \mathrm{mM}$ extracellular $\mathrm{Ca}^{2+}$ and Orai $1 \alpha$ serine phosphorylation was estimated $10 \mathrm{~s}, 1 \mathrm{~min}$ and $10 \mathrm{~min}$ after the addition of the agonist. As depicted in Fig. S6a, Western blot analysis reveals that Orai1 $\alpha$ serine phosphorylation was comparable in cells expressing TRPC1 and dnTRPC1 mutant, which indicates that $\mathrm{Ca}^{2+}$ influx via TRPC1 does not alter serine phosphorylation-dependent inactivation of Orai $\alpha$. We have further investigated whether TRPC1 might influence the plasma membrane expression of Orai $1 \alpha$ by biotinylation. HeLa cells were co-transfected with STIM1, Orai1 $\alpha$ and either TRPC1, dnTRPC1 or shTRPC1. Cells were stimulated with histamine $(3 \mu \mathrm{M})$ in the presence of $1 \mathrm{mM}$ extracellular $\mathrm{Ca}^{2+}$ and the exposure of Orai $1 \alpha$ in the plasma membrane was estimated $10 \mathrm{~s}, 1 \mathrm{~min}$ and $10 \mathrm{~min}$ after the addition of the agonist. As depicted in Fig. S6b, our results indicate that the plasma membrane location of Orai $1 \alpha$ was similar in cells expressing TRPC 1 or the dnTRPC 1 , as well as in cells transfected with shTRPC1, thus suggesting that TRPC1-mediated cation entry does not influence the plasma membrane expression of Orai $1 \alpha$. Altogether, these findings challenge a positive role for TRPC1 in $\mathrm{Ca}^{2+}$ influx 

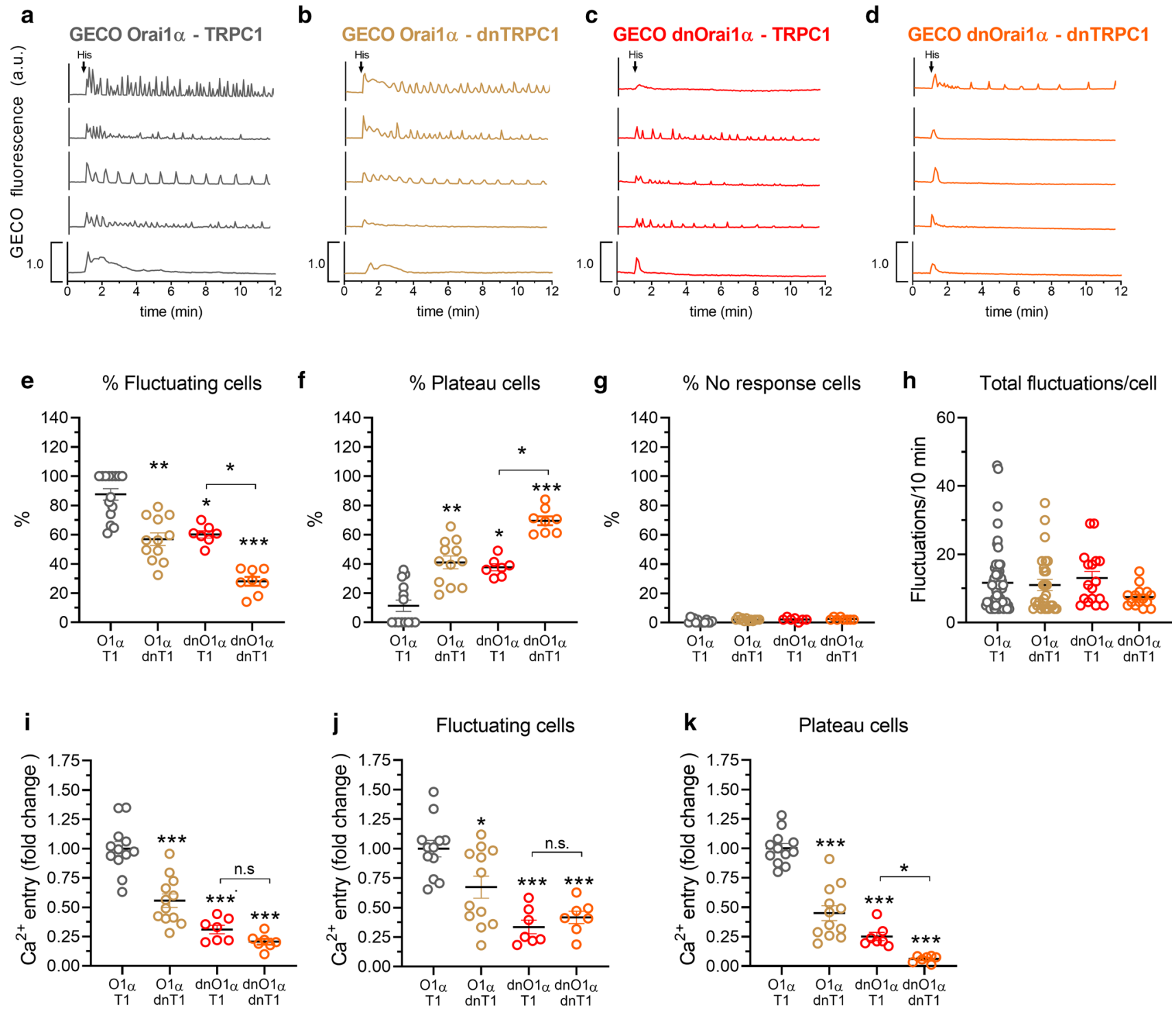

Fig. 3 TRPC1 modulates the function of Orai $1 \alpha$. a-d Representative $\mathrm{Ca}^{2+}$ responses to $3 \mu \mathrm{M}$ histamine measured using G-GECO1.2 in HeLa cells co-transfected with STIM1, G-GECO1.2-Orai1 $\alpha$ and TRPC1 or the corresponding dominant-negative mutants, as described. Cells were super-fused with HBSS containing $1 \mathrm{mM} \mathrm{Ca}^{2+}$ and stimulated with $3 \mu \mathrm{M}$ histamine at $1 \mathrm{~min}$ (indicated by arrow in a). Representative traces from five cells/condition were chosen to represent the datasets. $\mathbf{e}-\mathbf{h}$ Quantification of the percentage of fluctuating cells (e), percentage of plateau cells (f), percentage of non-responding

via Orai $1 \alpha$ and further support that Orai $1 \alpha$ and TRPC1 are in the close proximity.

To further explore whether $\mathrm{Ca}^{2+}$ entry via TRPC1 can influence G-GECO1.2 fluorescence, we repeated the experiments in cells expressing G-GECO fused to the dominant-negative Orai1 $\alpha$-E106Q mutant (GECOdnOrai1 $\alpha$ ), which lacks $\mathrm{Ca}^{2+}$ influx through the channel. It is noteworthy that under these conditions, TRPC 1 is not expected to be fully functional as $\mathrm{Ca}^{2+}$ entry through

cells (g) and total fluctuations/cell in $10 \mathrm{~min}(\mathbf{h})$ for data presented in a-d (for e-g, $n=7-14 ; n$ values correspond to independent experiments; for h, from left to right, $n=65,27,17$ and 15 ; $n$ values correspond to individual cells). $\mathbf{i}-\mathbf{k}$ Quantification of $\mathrm{Ca}^{2+}$ entry for all the conditions from a to d estimated in all the cells (i), fluctuating cells (j) and plateau cells (k). Scatter plots are represented as mean \pm SEM and were statistically analyzed using Kruskal-Wallis test with multiple comparisons (Dunn's test). $* p<0.05$ and $* * * p<0.001$ as compared to HeLa cells expressing G-GECO1.2-Orai $1 \alpha$ and TRPC1

Orai $1 \alpha$ is impaired [1]. Even with this limitation, when cells were co-transfected with STIM1, TRPC1 and GECO-dnOrai $1 \alpha$, the percentage of cells with fluctuations in G-GECO1.2 fluorescence and the magnitude of $\mathrm{Ca}^{2+}$ influx were significantly greater than in cells expressing dnTRPC1 $(p<0.05$; Fig. 3e-k). These findings indicate that $\mathrm{Ca}^{2+}$ entry via TRPC1 influences the fluorescence of G-GECO1.2 associated to Orai1 $\alpha$, which confirms that Orai $1 \alpha$ and TRPC 1 are adjacent. 


\section{Orai1 a modulates cation entry through TRPC1}

To investigate the possible role of Orai1 variants in the modulation of TRPC 1 function, HeLa cells were co-transfected with STIM1, TRPC1, Orai1 $\alpha$ and Orai1 $\beta$, or their respective dominant-negative mutants: dnOrai $1 \alpha$ and dnOrai $1 \beta . \mathrm{Mn}^{2+}$ was used to evaluate the effect of Orai $1 \alpha$ and Orai $1 \beta$ on TG-evoked bivalent-cation influx through TRPC1 as Orai1 has been reported to be poorly permeable to $\mathrm{Mn}^{2+}[36]$ and highly selective for $\mathrm{Ca}^{2+}$ in the presence of divalent cations [37]. This cation can be used as a surrogate for $\mathrm{Ca}^{2+}$ entry, given its quenching effect on fura-2 [38]. Cells were superfused with a medium containing $0.5 \mathrm{mM} \mathrm{Mn}^{2+}$ and $1 \mathrm{mM}$ $\mathrm{Ca}^{2+}$ and stimulated with $2 \mu \mathrm{M}$ TG. Fura- 2 was excited at the isoemissive wavelength, $360 \mathrm{~nm}$, to monitor the quenching of fluorescence by $\mathrm{Mn}^{2+}$. As shown in Fig. 4a, in cells cotransfected with STIM1, Orai $1 \alpha$, Orai $1 \beta$ and TRPC1, addition of TG resulted in a sustained quenching of fura- 2 fluorescence. Transient expression of STIM1, TRPC1, Orai $1 \alpha$ and Orai $1 \beta$ significantly enhanced TG-evoked quenching of fura-2 fluorescence as compared to mock-treated cells (Fig. S7). As shown in Fig. S8, co-expression of STIM1 and Orai $1 \alpha$ or Orai $\beta$ did not significantly enhance $\mathrm{Mn}^{2+}$ entry upon treatment with TG as compared to mock-treated cells, which indicates that $\mathrm{Mn}^{2+}$ enters the cell through TRPC1. Co-transfection of dnOrai $1 \alpha$, instead of WT Orai $1 \alpha$, resulted in an attenuation in the rate of $\mathrm{Mn}^{2+}$ entry (Fig. 4b). The initial rate of $\mathrm{Mn}^{2+}$-evoked fluorescence quenching in cells expressing dnOrai $1 \alpha$ was significantly decreased to approximately $50 \%$ of control (Fig. $4 \mathrm{e} ; p<0.001$ ). By contrast, cells co-expressing STIM1, TRPC1, Orai $\alpha$ and dnOrai1 $\beta$ exhibited a similar response to TG than those co-expressing Orai $1 \beta$ (Fig. $4 \mathrm{c}$ vs a), which strongly suggests that functional Orai1 $\beta$ is not required for TRPC1-mediated cation entry. Further substitution of both, Orai $1 \alpha$ and Orai $1 \beta$, for their respective pore-dead mutants significantly attenuated the rate of $\mathrm{Mn}^{2+}$ entry and the overall quenching of fura-2 fluorescence (Fig. 4d, e; $p<0.001$ ), although the effect of co-expression of both dominant-negative mutants was not found to be significantly different from that induced by dnOrail $\alpha$ (Fig. 4e). These findings indicate that Orai $1 \alpha$, but not Orai $1 \beta$, is required for TRPC 1 function.

We have noticed that our findings in HeLa cells differ from those reported by Desai et al. in HEK-293 cells, which showed that both Orai $1 \alpha$ - and Orai $1 \beta$-mediated signals could activate TRPC1 [15]. Therefore, we have repeated the experimental procedure used in HeLa cells to assess the role of Orai $1 \alpha$ and Orai $1 \beta$ on TRPC 1 activation in HEK-293 cells and we have found that, in these cells, co-expression of STIM1, Orai1 (EYFP-Orai1 plasmid, which might give Orai $1 \alpha$ and Orai1 $\beta$ ), and TRPC1 significantly enhances TG-evoked $\mathrm{Mn}^{2+}$ entry (Fig. S9a, b). This response was abolished when Orail was substituted by a dominant-negative Orai1 mutant (Fig. S9c).
As depicted in Fig. S9d-f, and in agreement with Desai et al. [15], both Orai $1 \alpha$ and Orai1 $\beta$ enhanced TG-evoked $\mathrm{Mn}^{2+}$ equally well, and generated signals comparable to those observed with wild-type Orai1, which confirms that, in HEK293 cells, Orai $1 \alpha$ and Orai $1 \beta$ are equally effective in activating TRPC1. Interestingly, these findings indicate intercellular differences in the functional role of Orai1 $\alpha$ and Orai1 $\beta$.

Next, we investigated the mechanism underlying the modulation of TRPC 1 by Orai $1 \alpha$ in HeLa cells. As TRPC 1 has been recruited to the PM by a mechanism dependent on Orai1 [1], we have explored whether the Orail variants play a relevant role in the surface exposure of TRPC1. HeLa cells were co-transfected with STIM1, TRPC1, Orai1 $\alpha$ and Orai $1 \beta$ or their respective dominant-negative mutants. In some experiments, cells were co-transfected with shTRPC1 instead of TRPC1 expression plasmid to assess specificity of the antibody. As shown in Fig. 4f, TRPC1 was clearly detected by biotinylation in the PM of cells expressing STIM1, TRPC1, Orai $1 \alpha$ and Orai $1 \beta$. Substitution of Orai $1 \alpha$ for dnOrai $1 \alpha$ significantly attenuated the amount of TRPC1 detected in the plasma membrane (Fig. 4f, g; $p<0.001$ ). Consistent with the results presented in Fig. 4e, co-expression of dnOrai $1 \beta$ instead of Orai $1 \beta$ did not significantly attenuate the expression of TRPC1 in the PM and substitution of both Orai1 variants for their respective dominantnegative mutants significantly attenuated TRPC1 exposure in the PM and led to results comparable to that observed in cells expressing dnOrai $1 \alpha$ (Fig. 4f, g; $p<0.01$ ). In cells transfected with shTRPC1, the expression of TRPC1 in the PM was undetectable (Fig. 4f).

We have further evaluated the role of Orai1 in TG-induced $\mathrm{Mn}^{2+}$ entry by TRPC1 in HeLa cells using the Orai1-specific inhibitor synta66. As shown in Fig. 5a, b, $\mathrm{Mn}^{2+}$ entry was significantly inhibited in cells treated with $10 \mu \mathrm{M}$ synta66 to a similar extent that in cells where TRPC1 has been knocked down, which further indicates that $\mathrm{Mn}^{2+}$ entry by TRPC1 requires functional Orail. We have further analyzed whether TRPC 1 activation by Orai $1 \alpha$ further depends on STIM1. To test this issue, HeLa cells were co-transfected with STIM1, Orai 1 and TRPC1 or with the STIM1(K684,685E) mutant (which is unable to activate TRPC1 $[39,40]$ ), Orai1 and TRPC1. As depicted in Fig. $5 \mathrm{c}, \mathrm{d}, \mathrm{Mn}^{2+}$ entry evoked by TG was unaltered in cells expressing the STIM1(K684,685E) mutant, thus suggesting that STIM1 is required for the activation of Orai1, but then, it does not seem to be further required to gate TRPC1 channels.

\section{Discussion}

Our present studies reveal that Orai $1 \alpha$ co-localizes with TRPC1 and is required for the location of this channel in the plasma membrane and its activation upon $\mathrm{Ca}^{2+}$ store 

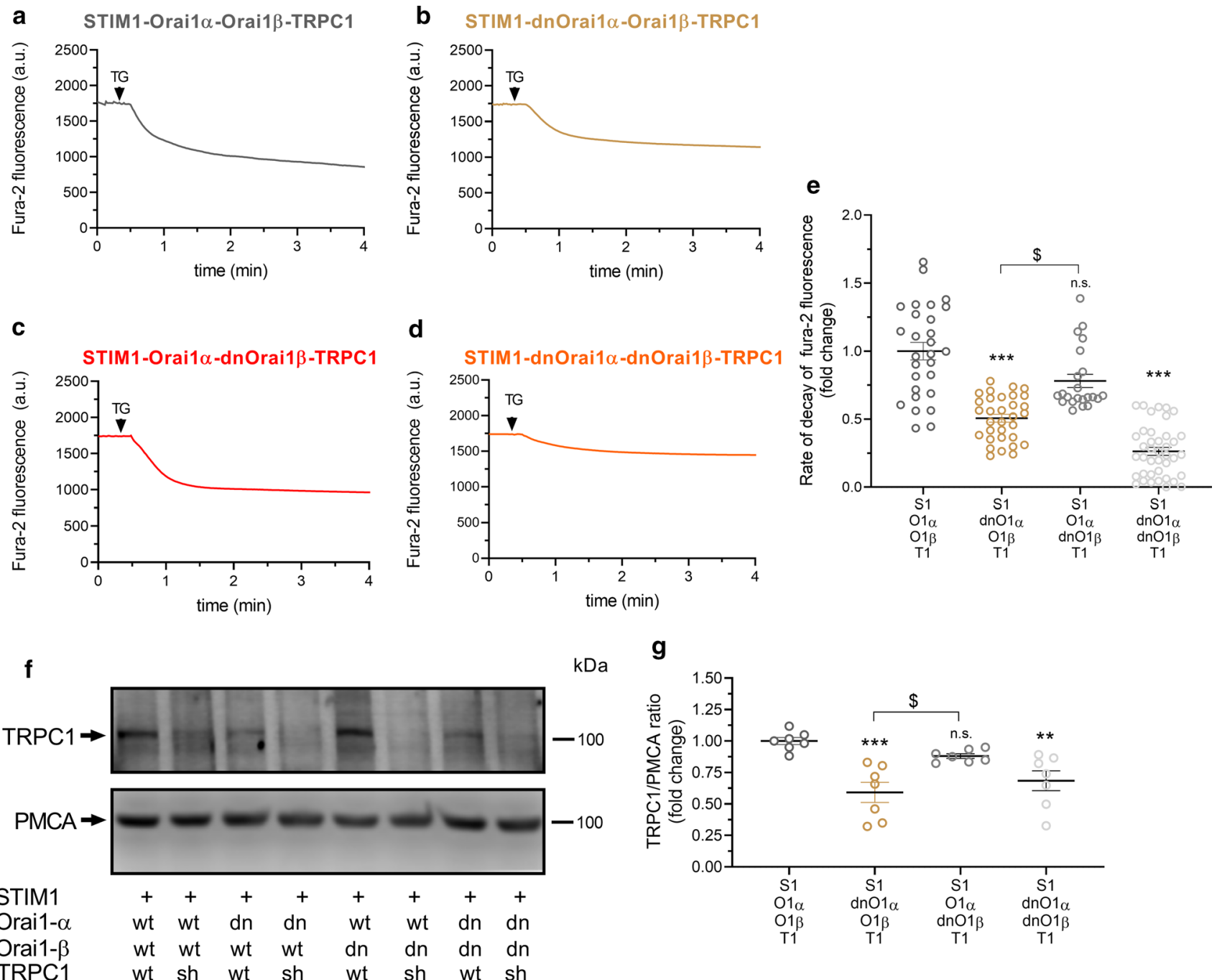

Fig. 4 Orai $1 \alpha$ modulates $\mathrm{Mn}^{2+}$ influx through TRPC1 and TRPC1 plasma membrane expression. a-d Representative responses to $2 \mu \mathrm{M}$ TG in HeLa cells co-transfected with STIM1, Orai1 $\alpha$, Orai1 $\beta$ and TRPC1 or the corresponding dominant-negative Orai1 mutants, as described. Cells were super-fused with HBSS containing $0.5 \mathrm{mM}$ $\mathrm{Mn}^{2+}$ and $1 \mathrm{mM} \mathrm{Ca}^{2+}$ and stimulated with $2 \mu \mathrm{M}$ TG (indicated by arrow). Fura-2 fluorescence was measured at an excitation wavelength of $360 \mathrm{~nm}$, the isoemissive wavelength. Representative traces were chosen to represent the datasets. e Quantification of the rate of decay of fura-2 fluorescence under the different experimental conditions (from left to right, $n=28,32,22$ and $40 ; n$ values correspond to individual cells). Scatter plots are represented as mean \pm SEM and were statistically analyzed using Kruskal-Wallis test with multiple comparisons (Dunn's test). ${ }^{* *} p<0.01$ and $* * * p<0.001$ as compared to HeLa cells expressing STIM1, Orai $1 \alpha$, Orai $1 \beta$ and TRPC1. ${ }^{\$} p<0.05$, statistical significance among the responses observed in cells expressing dnOrai $1 \alpha$ or dnOrai $1 \beta$ mutants. f HeLa cells were co-transfected with STIM1-CFP, Orai1 $\alpha$ (or dnOrai1 $\alpha$ mutant, as indicated), Orai1 $\beta$ (or dnOrai1 $\beta$ mutant, as indicated) and TRPC1

depletion or cell stimulation with physiological agonists. First, we have found interaction between Orai1 $\alpha$ and TRPC1 in native cells by co-immunoprecipitation using (or with shTRPC1, as indicated). Forty-eight hours later, cells were mixed with biotinylation buffer containing EZ-Link sulfo-NHS-LCbiotin, and cell surface proteins were labeled by biotinylation, as described in "Material and methods". Labeled proteins were pulled down with streptavidin-coated agarose beads. The pellet (containing the plasma membrane fraction) was analyzed by SDS-PAGE and Western blotting using anti-TRPC1 or anti-PMCA antibody, as indicated. Molecular masses indicated on the right were determined using molecular-mass markers run in the same gel. These results are representative of seven separate experiments. g Quantification of TRPC1 plasma membrane expression under the different experimental conditions normalized to the PMCA expression. Scatter plots are represented as mean \pm SEM and were statistically analyzed using KruskalWallis test with multiple comparisons (Dunn's test). $* * p<0.01$ and $* * * p<0.001$ as compared to HeLa cells expressing STIM1, Orai $1 \alpha$, Orai1 $\beta$ and TRPC1. ${ }^{\$} p<0.05$, statistical significance among the TRPC1 surface expression in cells expressing dnOrai1 $\alpha$ or dnOrai $1 \beta$ mutants

both anti-Orai1 and anti-TRPC1 antibodies. Second, as antibody-based approaches strongly depend on the specificity of the antibodies themselves, we have further demonstrated 

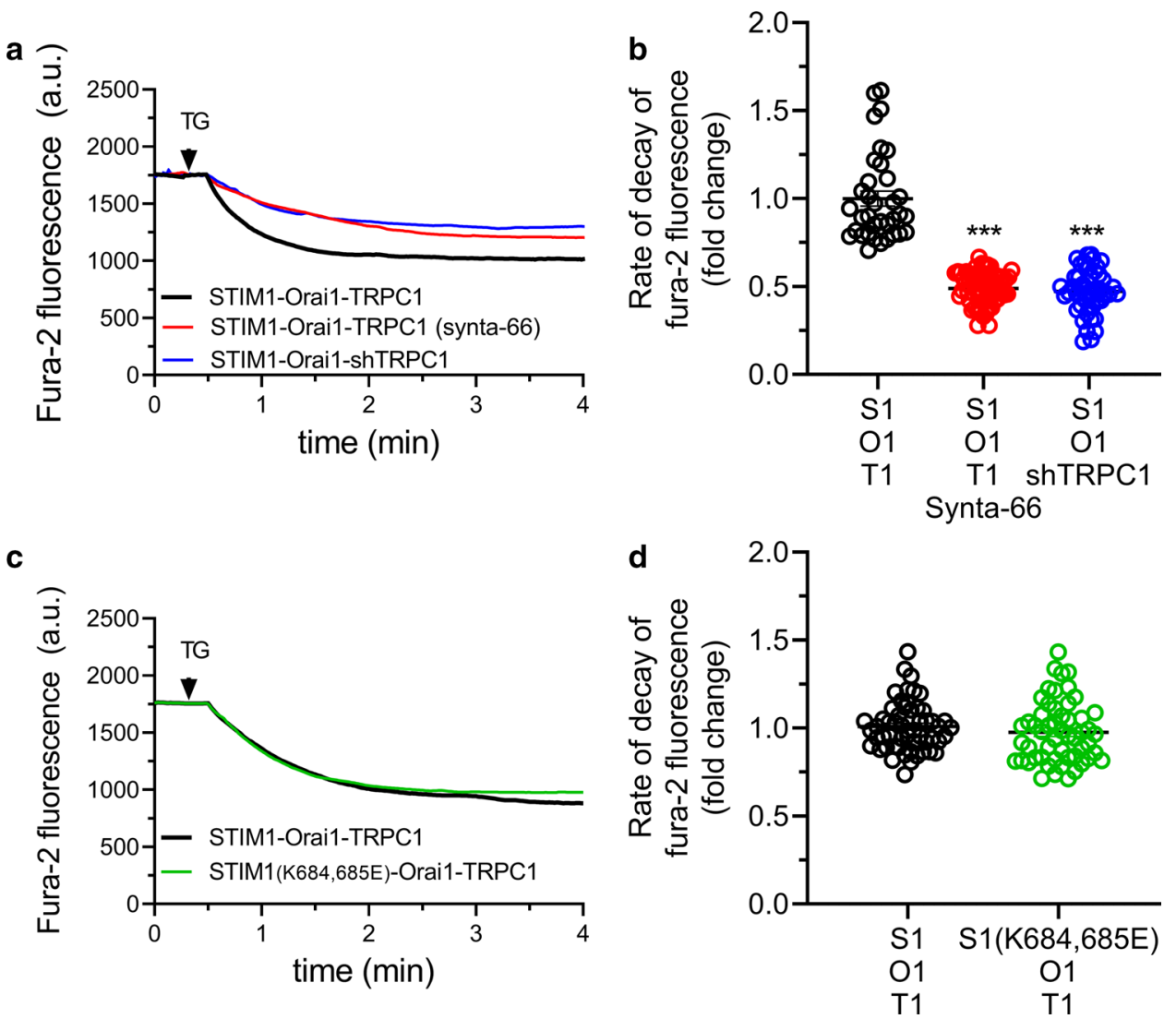

Fig. 5 Effect of Orail inhibition and expression of the STIM1 (K684,685E) mutant on $\mathrm{Mn}^{2+}$ influx in HeLa cells. a Representative responses to $2 \mu \mathrm{M}$ TG in HeLa cells co-transfected with STIM1, Orai1 and TRPC1, in the absence or presence of synta66 (10 $\mu \mathrm{M})$, or co-transfected with STIM1, Orai1 and shTRPC1, as described. Cells were super-fused with HBSS containing $0.5 \mathrm{mM} \mathrm{Mn}^{2+}$ and $1 \mathrm{mM}$ $\mathrm{Ca}^{2+}$ and stimulated with $2 \mu \mathrm{M}$ TG (indicated by arrow). Fura-2 fluorescence was measured at an excitation wavelength of $360 \mathrm{~nm}$, the isoemissive wavelength. Representative traces were chosen to represent the datasets. b Quantification of the rate of decay of fura-2 fluorescence under the different experimental conditions (from left to right, $n=35,61$ and $47 ; n$ values correspond to individual cells). Scatter plots are represented as mean \pm SEM and were statistically analyzed using Kruskal-Wallis test with multiple comparisons

co-localization of Orai1 $\alpha$ and TRPC1 by an independent mechanism using APEX2 proximity labeling assay in cells expressing STIM1-CFP, TRPC1 and Orai1 $\alpha$-GFP. These approaches indicate co-localization of Orai1 $\alpha$ and TRPC1 under resting conditions and that this interaction significantly enhances upon $\mathrm{Ca}^{2+}$ store depletion using TG or histamine. Nevertheless, our findings suggest that Orai $1 \beta$ is scarcely associated to TRPC1 under resting conditions but this interaction, if significant, is constitutive and does not depend on $\mathrm{Ca}^{2+}$ store depletion or stimulation with agonists.

Our results provide further evidence for the co-localization between Orai $1 \alpha$ and TRPC 1 by revealing cross-detection of TRPC1-mediated $\mathrm{Ca}^{2+}$ entry by G-GECO-Orai1 $\alpha$. GECO is a channel-fused fluorescent $\mathrm{Ca}^{2+}$ indicator that
(Dunn's test). $* * * p<0.001$ as compared to HeLa cells expressing STIM1, Orail and TRPC1. c Representative responses to TG in HeLa cells co-transfected with STIM1 or the STIM1(K684,685E) mutant, Orai1 and TRPC1, as indicated. Cells were super-fused with HBSS containing $0.5 \mathrm{mM} \mathrm{Mn}^{2+}$ and $1 \mathrm{mM} \mathrm{Ca}^{2+}$ and stimulated with $2 \mu \mathrm{M}$ TG (indicated by arrow). Fura-2 fluorescence was measured at an excitation wavelength of $360 \mathrm{~nm}$, the isoemissive wavelength. Representative traces were chosen to represent the datasets. d Quantification of the rate of decay of fura-2 fluorescence under the different experimental conditions (from left to right, $n=55$ and $54 ; n$ values correspond to individual cells). Scatter plots are represented as mean \pm SEM and were statistically analyzed using Mann-Whitney $U$ test

detects changes in $\mathrm{Ca}^{2+}$ concentration in the close proximity of the channel to which it is associated [41]. We have previously found that G-GECO-Orai $1 \alpha$ is unable to detect changes in the $\mathrm{Ca}^{2+}$ concentration near the channel upon discharge of the endoplasmic reticulum using TG in HeLa cells [30], thus suggesting that TRPC1 should be close enough to Orai1 $\alpha$ to influence the signal of the fluorophore. Altogether, using three different experimental approaches, our results provide strong evidence for the co-localization of Orail $\alpha$ and TRPC1, an event that is regulated by $\mathrm{Ca}^{2+}$ store depletion or stimulation with physiological agonists.

An additional important finding of our study is that TRPC1 plasma membrane location and function is strongly dependent on the functional expression of Orai $1 \alpha$, as 
expression of the dominant-negative Orai $1 \alpha$ mutant (Orai $1 \alpha$ E106Q-EGFP), instead of functional Orai $1 \alpha$, significantly impaired both the surface exposure of TRPC 1 and divalent cation entry through the channel. In support of these findings, inhibition of Orail by synta66 significantly attenuated $\mathrm{Mn}^{2+}$ entry to a similar extent to TRPC1 knockdown in HeLa cells. The experiments were performed in the presence of $1 \mathrm{mM}$ extracellular $\mathrm{Ca}^{2+}$ to maintain Orail $\mathrm{Ca}^{2+}$ selectivity [8] and allow Orai1-mediated $\mathrm{Ca}^{2+}$ entry-dependent responses, such as TRPC1 expression in the plasma membrane [1]. Consistent with this observation, in HeLa cells co-expressing dnOrai $1 \alpha$, the magnitude of histamine-evoked $\mathrm{Ca}^{2+}$ responses was similar either expressing TRPC1 or the dominant-negative TRPC1 mutant (see Fig. 1), which further confirms that functional Orai $1 \alpha$ is essential for TRPC1 channel function. By contrast, our results indicate that the short Orai1 variant, Orai1 $\beta$, has a negligible effect, if any, on the plasma membrane location and function of TRPC1. These findings are consistent with previous studies reporting that location of TRPC1 in the plasma membrane depends on $\mathrm{Ca}^{2+}$ influx through Orai1 [26] and further identifies the Orai1 variant involved in this process. Furthermore, this observation reveals important functional differences between both Orai 1 variants. The role of Orai $1 \alpha$, but not Orai $1 \beta$, in TRPC1 plasma membrane expression in HeLa cells might be associated to the presence exclusively in Orai1 $\alpha$ of a caveolin-binding domain, which allows Orail $\alpha$ interact to caveolin [42]. Caveolin-1 has also been reported to mediate trafficking of TRPC1 to the plasma membrane, which may be required for SOCE [43]. As TRPC1 trafficking to the plasma membrane requires $\mathrm{Ca}^{2+}$ entry via Orai1 [44] and both Orai $1 \alpha$ and TRPC1 co-localize with caveolin-1, it is expected that Orai $1 \alpha$ plays a predominant role triggering TRPC1 trafficking to the plasma membrane in HeLa cells. In addition to the caveolin-binding domain, other $\mathrm{N}$-terminal functional motifs of Orai $1 \alpha$, missing in Orai $1 \beta$, might lead to the recruitment of TRPC1 in the plasma membrane by Orai $1 \alpha$, presumably in the lipid rafts, where TRPC 1 has been located [45]. For instance, the AC8-binding domain, which is responsible for the interaction of Orai $1 \alpha$ with AC8 in lipid raft domains $[46,47]$ might play a relevant role in the location and recruitment of signaling proteins associated to the Orai $\alpha$ channelosome, including TRPC1.

Upon stimulation with physiological concentrations of agonists, cells develop repetitive oscillations in the concentration of cytosolic $\mathrm{Ca}^{2+}$ whose frequency and intensity have been reported to play a key role in the development of a number of cellular events, such as gene transcription [48]. $\mathrm{Ca}^{2+}$ oscillations are triggered by $\mathrm{IP}_{3}$-evoked $\mathrm{Ca}^{2+}$ release from the intracellular $\mathrm{Ca}^{2+}$ stores leading to the activation of store-operated channels in the plasma membrane [5, 11]. Using a four-fold co-expression of STIM1, Orai $1 \alpha$, Orai $1 \beta$ and TRPC 1 or a triple co-expression combination of STIM1, Orai $1 \alpha$ or Orai $1 \beta$ and TRPC1, we provide evidence supporting that both, Orai1 $\alpha$ and Orai $1 \beta$, are required to sustain $\mathrm{Ca}^{2+}$ oscillations, while TRPC1 has a minor role, if any. While TRPC1 does not seem to play a relevant role in $\mathrm{Ca}^{2+}$ oscillations, recruitment of this channel clearly enhances the magnitude of $\mathrm{Ca}^{2+}$ responses (observed in cells expressing functional Orai $1 \alpha$ (see Figs. $1 \mathrm{~m}$ and $\mathrm{S} 4 \mathrm{~m}$ ), which further confirms that TRPC1 function depends on $\mathrm{Ca}^{2+}$ influx via Orai1 $\alpha$ ). The observation that TRPC1 is not required for $\mathrm{Ca}^{2+}$ oscillations cannot be attributed to a loss of function of transiently expressed TRPC1 due to ectopic location or any other artifact as co-expression of STIM1, Orai $1 \alpha$, Orai $1 \beta$ and TRPC 1 in HeLa cells resulted in a robust $\mathrm{Mn}^{2+}$ entry upon stimulation with TG in the presence of extracellular $\mathrm{Ca}^{2+}$.

In conclusion, our results provide multiple evidence for store depletion-dependent co-localization of Orai $1 \alpha$ with TRPC1 and the regulation of TRPC1 plasma membrane expression and channel function by Orai $1 \alpha$; by contrast, the short Orai1 variant, Orai1 $\beta$, is not required for plasma membrane expression and function of TRPC1. The approaches used do not allow sufficient resolution to discriminate if Orai1 $\alpha$ and TRPC1 interact directly or they are located in the same cellular nano-domain as near components of a functional protein complex. Our results provide evidence that Orai $1 \alpha$ and Orai1 $\beta$ are non-redundant and might display differential functional roles in calcium signaling.

Supplementary Information The online version contains supplementary material available at https://doi.org/10.1007/s00018-021-04098-w.

Acknowledgements We thank Sandra Alvarado, Alvaro Macías and Diego Mena for technical assistance in some experiments.

Author contributions Conceptualization: JAR, JJL, TS, GMS. Methodology: JS-C, JJL. Investigation: JS-C, JJL, AB-E, CC, PJC, IJ. Supervision: JAR, TS, GMS. Writing—original draft: JAR, JJL, JS-C. Writing-review and editing: IJ, PJC, AB-E, TS, GMS.

Funding Open Access funding provided thanks to the CRUE-CSIC agreement with Springer Nature. Grants PID2019-104084 GB-C21 and PID2019-104084 GB-C22 funded by MCIN/AEI/ https://doi.org/10. $13039 / 501100011033$ and ERDF A way of making Europe, and Junta de Extremadura-Fondo Europeo de Desarrollo Regional (FEDER; Grants IB20007, IB18025, GR21008 and GR18061) to JAR and TS. JJL and IJ are supported by a contract from Junta de Extremadura (TA18011 and TA18054, respectively). JS-C is supported by a contract from Ministry of Science, Innovation, and Universities, Spain. CC is supported by a Predoctoral fellowship of the Junta de Extremadura (PD16072). AB-E is supported by Junta de Extremadura (DPCI0643).

Data availability All data are available in the main text or the supplementary materials. 


\section{Declarations}

Conflict of interest The authors declare no conflict of interest.

Ethical approval Experimental procedures were approved by the local ethical committee (University of Extremadura and Extremadura Health Service).

Open Access This article is licensed under a Creative Commons Attribution 4.0 International License, which permits use, sharing, adaptation, distribution and reproduction in any medium or format, as long as you give appropriate credit to the original author(s) and the source, provide a link to the Creative Commons licence, and indicate if changes were made. The images or other third party material in this article are included in the article's Creative Commons licence, unless indicated otherwise in a credit line to the material. If material is not included in the article's Creative Commons licence and your intended use is not permitted by statutory regulation or exceeds the permitted use, you will need to obtain permission directly from the copyright holder. To view a copy of this licence, visit http://creativecommons.org/licenses/by/4.0/.

\section{References}

1. Ong HL, Subedi KP, Son GY, Liu X, Ambudkar IS (2019) Tuning store-operated calcium entry to modulate $\mathrm{Ca}^{2+}$-dependent physiological processes. Biochim Biophys Acta Mol Cell Res 1866(7):1037-1045

2. Berridge MJ, Lipp P, Bootman MD (2000) The versatility and universality of calcium signalling. Nat Rev Mol Cell Biol 1(1):11-21

3. Kar P, Lin YP, Bhardwaj R, Tucker CJ, Bird GS, Hediger MA et al (2021) The N terminus of Orai1 couples to the AKAP79 signaling complex to drive NFAT1 activation by local $\mathrm{Ca}^{2+}$ entry. Proc Natl Acad Sci USA 118(19):e2012908118

4. Zhang X, Pathak T, Yoast R, Emrich S, Xin P, Nwokonko RM et al (2019) A calcium/cAMP signaling loop at the ORAI1 mouth drives channel inactivation to shape NFAT induction. Nat Commun 10(1):1971

5. Kar P, Bakowski D, Di Capite J, Nelson C, Parekh AB (2012) Different agonists recruit different stromal interaction molecule proteins to support cytoplasmic $\mathrm{Ca}^{2+}$ oscillations and gene expression. Proc Natl Acad Sci USA 109(18):6969-6974

6. Roos J, DiGregorio PJ, Yeromin AV, Ohlsen K, Lioudyno M, Zhang S et al (2005) STIM1, an essential and conserved component of store-operated $\mathrm{Ca}^{2+}$ channel function. J Cell Biol 169(3):435-445

7. Zhang SL, Yu Y, Roos J, Kozak JA, Deerinck TJ, Ellisman MH et al (2005) STIM1 is a $\mathrm{Ca}^{2+}$ sensor that activates CRAC channels and migrates from the $\mathrm{Ca}^{2+}$ store to the plasma membrane. Nature 437(7060):902-905

8. Mercer JC, Dehaven WI, Smyth JT, Wedel B, Boyles RR, Bird GS et al (2006) Large store-operated calcium selective currents due to co-expression of Orai1 or Orai2 with the intracellular calcium sensor, Stim1. J Biol Chem 281(34):24979-24990

9. Peinelt C, Vig M, Koomoa DL, Beck A, Nadler MJ, KoblanHuberson $M$ et al (2006) Amplification of CRAC current by STIM1 and CRACM1 (Orai1). Nat Cell Biol 8(7):771-773

10. Prakriya M, Feske S, Gwack Y, Srikanth S, Rao A, Hogan PG (2006) Orai1 is an essential pore subunit of the CRAC channel. Nature 443(7108):230-233

11. Emrich SM, Yoast RE, Xin P, Arige V, Wagner LE, Hempel N et al (2021) Omnitemporal choreographies of all five STIM/Orai and IP3Rs underlie the complexity of mammalian $\mathrm{Ca}^{2+}$ signaling. Cell Rep 34(9):108760

12. Schindl R, Frischauf I, Bergsmann J, Muik M, Derler I, Lackner $\mathrm{B}$ et al (2009) Plasticity in $\mathrm{Ca}^{2+}$ selectivity of Orai1/Orai3 heteromeric channel. Proc Natl Acad Sci USA 106(46):19623-19628

13. Yoast RE, Emrich SM, Zhang X, Xin P, Johnson MT, Fike AJ et al (2020) The native ORAI channel trio underlies the diversity of $\mathrm{Ca}^{2+}$ signaling events. Nat Commun 11(1):2444

14. Grabmayr H, Romanin C, Fahrner M (2020) STIM proteins: an ever-expanding family. Int J Mol Sci 22(1):378

15. Desai PN, Zhang X, Wu S, Janoshazi A, Bolimuntha S, Putney JW et al (2015) Multiple types of calcium channels arising from alternative translation initiation of the Orail message. Sci Signal 8(387):ra74

16. Ambudkar IS, de Souza LB, Ong HL (2017) TRPC1, Orai1, and STIM1 in SOCE: Friends in tight spaces. Cell Calcium 63:33-39

17. Parekh AB, Putney JW Jr (2005) Store-operated calcium channels. Physiol Rev 85(2):757-810

18. Ong HL, Ambudkar IS (2017) STIM-TRP pathways and microdomain organization: contribution of TRPC1 in store-operated $\mathrm{Ca}(2+)$ entry: impact on $\mathrm{Ca}(2+)$ signaling and cell function. Adv Exp Med Biol 993:159-188

19. Putney JW Jr (2007) Inositol lipids and TRPC channel activation. Biochem Soc Symp 74:37-45

20. Ong HL, de Souza LB, Cheng KT, Ambudkar IS (2014) Physiological functions and regulation of TRPC channels. Handb Exp Pharmacol 223:1005-1034

21. Cheng KT, Liu X, Ong HL, Ambudkar IS (2008) Functional requirement for Orai1 in store-operated TRPC1-STIM1 channels. J Biol Chem 283(19):12935-12940

22. Shi J, Miralles F, Birnbaumer L, Large WA, Albert AP (2016) Store depletion induces Galphaq-mediated PLCbeta1 activity to stimulate TRPC1 channels in vascular smooth muscle cells. Faseb J 30(2):702-715

23. Shi J, Miralles F, Kinet JP, Birnbaumer L, Large WA, Albert AP (2017) Evidence that Orail does not contribute to storeoperated TRPC1 channels in vascular smooth muscle cells. Channels (Austin) 11(4):329-339

24. Avila-Medina J, Calderon-Sanchez E, Gonzalez-Rodriguez P, Monje-Quiroga F, Rosado JA, Castellano A et al (2016) Orai1 and TRPC1 proteins co-localize with $\mathrm{CaV} 1.2$ channels to form a signal complex in vascular smooth muscle cells. J Biol Chem 291(40):21148-21159

25. Jardin I, Lopez JJ, Salido GM, Rosado JA (2008) Orai1 mediates the interaction between STIM1 and hTRPC1 and regulates the mode of activation of hTRPC1-forming $\mathrm{Ca}^{2+}$ channels. J Biol Chem 283(37):25296-25304

26. Cheng KT, Liu X, Ong HL, Swaim W, Ambudkar IS (2011) Local $\mathrm{Ca}^{2+}$ entry via Orai1 regulates plasma membrane recruitment of TRPC1 and controls cytosolic $\mathrm{Ca}^{2+}$ signals required for specific cell functions. PLoS Biol 9(3):e1001025

27. Shalygin A, Kolesnikov D, Glushankova L, Gusev K, Skopin A, Skobeleva K et al (2021) Role of STIM2 and Orai proteins in regulating TRPC 1 channel activity upon calcium store depletion. Cell Calcium 97:102432

28. Fukushima M, Tomita T, Janoshazi A, Putney JW (2012) Alternative translation initiation gives rise to two isoforms of Orai1 with distinct plasma membrane mobilities. J Cell Sci $125(\mathrm{Pt}$ 18):4354-4361

29. Albarran L, Lopez JJ, Jardin I, Sanchez-Collado J, Berna-Erro A, Smani T et al (2018) EFHB is a novel cytosolic $\mathrm{Ca}^{2+}$ sensor that modulates STIM1-SARAF interaction. Cell Physiol Biochem 51(3):1164-1178

30. Sanchez-Collado J, Lopez JJ, Jardin I, Camello PJ, Falcon D, Regodon S et al (2019) Adenylyl cyclase type 8 overexpression impairs phosphorylation-dependent Orai1 inactivation and 
promotes migration in MDA-MB-231 breast cancer cells. Cancers (Basel) 11(11):1624

31. Tan B, Peng S, Yatim S, Gunaratne J, Hunziker W, Ludwig A (2020) An optimized protocol for proximity biotinylation in confluent epithelial cell cultures using the peroxidase APEX2. STAR Protocols 1(2):100074

32. Jardin I, Lopez JJ, Redondo PC, Salido GM, Rosado JA (2009) Store-operated $\mathrm{Ca}^{2+}$ entry is sensitive to the extracellular $\mathrm{Ca}^{2+}$ concentration through plasma membrane STIM1. Biochim Biophys Acta 1793(10):1614-1622

33. Sanchez-Collado J, Lopez JJ, Gonzalez-Gutierrez L, Cantonero C, Jardin I, Salido GM et al (2020) Functional role of TRPC6 and STIM2 in cytosolic and endoplasmic reticulum $\mathrm{Ca}^{2+}$ content in resting estrogen receptor-positive breast cancer cells. Biochem J 477(17):3183-3197

34. Saul S, Stanisz H, Backes CS, Schwarz EC, Hoth M (2014) How ORAI and TRP channels interfere with each other: interaction models and examples from the immune system and the skin. Eur J Pharmacol 739:49-59

35. Kawasaki T, Ueyama T, Lange I, Feske S, Saito N (2010) Protein kinase $\mathrm{C}$-induced phosphorylation of Orai1 regulates the intracellular $\mathrm{Ca}^{2+}$ level via the store-operated $\mathrm{Ca}^{2+}$ channel. J Biol Chem 285(33):25720-25730

36. Bulla M, Gyimesi G, Kim JH, Bhardwaj R, Hediger MA, Frieden $\mathrm{M}$ et al (2019) ORAI1 channel gating and selectivity is differentially altered by natural mutations in the first or third transmembrane domain. J Physiol 597(2):561-582

37. Muik M, Schindl R, Fahrner M, Romanin C (2012) $\mathrm{Ca}^{2+}$ releaseactivated $\mathrm{Ca}^{2+}$ (CRAC) current, structure, and function. Cell Mol Life Sci 69(24):4163-4176

38. Rosado JA, Sage SO (2000) Farnesylcysteine analogues inhibit store-regulated $\mathrm{Ca}^{2+}$ entry in human platelets: evidence for involvement of small GTP-binding proteins and actin cytoskeleton. Biochem J 347(Pt 1):183-192

39. Zeng W, Yuan JP, Kim MS, Choi YJ, Huang GN, Worley PF, Muallem S (2008) STIM1 gates TRPC channels, but not Orai1, by electrostatic interaction. Mol Cell 32(3):439-448

40. Jardin I, Dionisio N, Frischauf I, Berna-Erro A, Woodard GE, López JJ, Salido GM, Rosado JA (2013) The polybasic lysine-rich domain of plasma membrane-resident STIM1 is essential for the modulation of store-operated divalent cation entry by extracellular calcium. Cell Signal 25(5):1328-1337

41. Dynes JL, Amcheslavsky A, Cahalan MD (2016) Genetically targeted single-channel optical recording reveals multiple Orai1 gating states and oscillations in calcium influx. Proc Natl Acad Sci USA 113(2):440-445

42. Yu F, Sun L, Machaca K (2010) Constitutive recycling of the store-operated $\mathrm{Ca}^{2+}$ channel Orail and its internalization during meiosis. J Cell Biol 191(3):523-535

43. Lockwich TP, Liu X, Singh BB, Jadlowiec J, Weiland S, Ambudkar IS (2000) Assembly of Trp1 in a signaling complex associated with caveolin-scaffolding lipid raft domains. J Biol Chem 275(16):11934-11942

44. de Souza LB, Ong HL, Liu X, Ambudkar IS (2015) Fast endocytic recycling determines TRPC1-STIM1 clustering in ER-PM junctions and plasma membrane function of the channel. Biochim Biophys Acta 1853(10 Pt A):2709-2721

45. Ong HL, Ambudkar IS (2011) The dynamic complexity of the TRPC1 channelosome. Channels 5(5):424-431

46. Tabbasum VG, Cooper DMF (2019) Structural and functional determinants of AC8 trafficking, targeting and responsiveness in lipid raft microdomains. J Membr Biol 252(2-3):159-172

47. Willoughby D, Everett KL, Halls ML, Pacheco J, Skroblin P, Vaca L, Klussmann E, Cooper DM (2012) Direct binding between Orai1 and AC8 mediates dynamic interplay between $\mathrm{Ca}^{2+}$ and cAMP signaling. Sci Signal 5(219):ra9

48. Dolmetsch RE, Xu K, Lewis RS (1998) Calcium oscillations increase the efficiency and specificity of gene expression. Nature 392(6679):933-936

Publisher's Note Springer Nature remains neutral with regard to jurisdictional claims in published maps and institutional affiliations. 